\title{
ASYMPTOTICS OF SOME ULTRA-SPHERICAL POLYNOMIALS AND THEIR EXTREMA*
}

\author{
MAGALI RIBOT ${ }^{\dagger}$
}

\begin{abstract}
Motivated by questions on the preconditioning of spectral methods, and independently of the extensive literature on the approximation of zeroes of orthogonal polynomials, either by the Sturm method, or by the descent method, we develop a stationary phase-like technique for calculating asymptotics of Legendre polynomials. The difference with the classical stationary phase method is that the phase is a nonlinear function of the large parameter and the integration variable, instead of being a product of the large parameter by a function of the integration variable. We then use an implicit functions theorem for approximating the zeroes of the derivatives of Legendre polynomials. This result is used for proving order and consistency of the residual smoothing scheme [1], [19].
\end{abstract}

Key words. ultra-spherical polynomials, stationary phase method, spectral methods, preconditioning, finite elements methods

AMS subject classifications. 33C45, 65N35(65N30)

1. Introduction. When we discretize implicitly in time a partial differential equation, we have to solve a linear system, where the matrix depends on the method used for the spatial discretization. Spectral methods are classical methods, but they produce matrices, which are not sparse and difficult to invert; therefore, their numerical efficiency depends on the introduction of appropriate preconditioners. A preconditioner $P$ of a matrix $M$ is a matrix, which can be more easily inverted than $M$ and such that the condition number of $P^{-1} M$, that is to say the product of the norm of the matrix $P^{-1} M$ by the norm of its inverse $M^{-1} P$, is as close to 1 as possible.

In the case of a Laplace - or more generally an elliptic - operator, finite differences or finite elements methods have been proposed for preconditioning spectral methods in Orszag [13], Haldenwang et al. [11], Canuto and Quarteroni [3] or Deville and Mund $[7,8]$.

In [18], Quarteroni and Zampieri investigate the finite element preconditioning of Legendre spectral methods for various boundary conditions; in this article, they show numerical evidence of the spectral equivalence between the Legendre spectral matrix and the finite element matrix. They also apply the preconditioner they propose to domain decomposition methods in the framework of the elasticity problem.

Let us briefly recall that in the one-dimensional situation of a Laplace operator, the coefficients of the mass matrix are defined by the scalar product of the elements of the basis, whereas the coefficients of the stiffness matrix are given by the scalar product of the derivatives of the elements of the basis.

Denote by $K_{S}$ the stiffness matrix associated to a spectral Legendre-GaussLobatto method for $-d^{2} / d x^{2}$ with Dirichlet boundary conditions, and by $K_{F}$ the stiffness matrix associated to the $P_{1}$ finite elements method on the nodes of this spectral method.

\footnotetext{
*Received April 16, 2004; accepted for publication June 15, 2005.

$\dagger$ Laboratoire Dieudonné, Université de Nice-Sophia Antipolis, 06108 Nice Cedex 2, France (ribot @math.unice.fr). I would like to thank very warmly Michelle Schatzman for pointing me out this subject and for many helpful discussions. Many thanks are due to Seymour Parter and David Gottlieb for their generous advice and encouragements.
} 
Let $M_{S}$ be the mass matrix of the spectral method and let $M_{F}$ be the masslumped matrix of the $P_{1}$ finite elements method constructed on the nodes of the spectral method. We define precisely all these matrices in [19]. We only need here the coefficients of the diagonal matrix $M_{F}^{-1} M_{S}$, which are given later in formula (1.6).

Recent results of Parter [15] give the following bounds:

$$
\frac{1}{C} \leq \frac{\operatorname{Re}\left(K_{F} M_{S} M_{F}^{-1} U, U\right)}{\left(K_{S} U, U\right)} \leq \frac{\left|\left(K_{F} M_{S} M_{F}^{-1} U, U\right)\right|}{\left(K_{S} U, U\right)} \leq C .
$$

Here (, ) denotes the canonical Hermitian scalar product. These results are based on [14], which itself builds on Gatteschi's results [9]. When $M_{F}$ is not mass-lumped, Parter [16] proves an analogous result to estimates (1.1).

The main result of [19] is the spectral equivalence between

$$
M_{S}^{1 / 2} M_{F}^{-1 / 2} K_{F} M_{F}^{-1 / 2} M_{S}^{1 / 2}
$$

and $K_{S}$. As a consequence of a result of Parter and Rothman [17], which says that $K_{F}$ and $K_{S}$ are equivalent, it suffices to prove the spectral equivalence between $K_{F}$ and

$$
M_{S}^{1 / 2} M_{F}^{-1 / 2} K_{F} M_{F}^{-1 / 2} M_{S}^{1 / 2} .
$$

This question is motivated by the analysis of the residual smoothing scheme (see [1] and [19]), which allows for fast time integration of the spectral approximation of parabolic equation.

It turns out that when I started working on this question, I was not aware of Parter's results, and I did not consult the recent literature on orthogonal polynomials; instead of using a Sturm method or a descent method, as is done by most authors in this field, I took the classical integral representation formula for ultra-spherical polynomials (4.10.3) from Szegö [20], and I applied to this formula a stationary phase strategy, in a region where the classical expansions cannot be applied; this method gives an expansion at all orders, with estimates for the error bound. Let us point out that this is not a classical stationary phase method, since the exponential term is a non linear function of the large parameter and of the integration variable.

Though the present result on preconditioning can be obtained with Parter's method, I feel that the treatment presented here of the asymptotics is novel and more general. Indeed, the detailed calculations given here for derivatives of Legendre polynomials could possibly be generalized to other classes of orthogonal polynomials, such as derivatives of Chebyshev polynomials or more generally to all ultra-spherical polynomials.

Let us describe why we need precise asymptotics of the zeroes of the derivatives of Legendre polynomials to prove the equivalence between $M_{S}^{1 / 2} M_{F}^{-1 / 2} K_{F} M_{F}^{-1 / 2} M_{S}^{1 / 2}$ and $K_{F}$. Let us also define precisely our notations.

We denote by $\mathbb{P}_{N}$ the space of polynomial functions of degree $N$ defined over $[-1,1]$. Let us denote by $L_{N}$ the Legendre polynomial of degree $N$ and let $-1=$ $\xi_{0}<\xi_{1}<\cdots<\xi_{N-1}<\xi_{N}=1$ be the roots of $\left(1-X^{2}\right) L_{N}^{\prime}$; they are the nodes of the spectral method. Let $\rho_{k}, 0 \leq k \leq N$ be the weights of the quadrature formula associated to the nodes $\xi_{k}$; since this is a Gauss-Lobatto formula, we shall have

$$
\forall \Phi \in \mathbb{P}_{2 N-1}, \int_{-1}^{1} \Phi(x) d x=\sum_{k=0}^{N} \Phi\left(\xi_{k}\right) \rho_{k} ;
$$


the weights $\rho_{k}$ are strictly positive.

Bernardi and Maday [2] give explicit expressions of the $\rho_{k}$ 's:

$$
\begin{aligned}
& \rho_{0}=\rho_{N}=\frac{2}{N(N+1)}, \\
& \rho_{k}=\frac{2}{N(N+1) L_{N}^{2}\left(\xi_{k}\right)}, 1 \leq k \leq N-1 .
\end{aligned}
$$

We define $\eta_{k}$ by

$$
\eta_{k}=\operatorname{Arccos}\left(\xi_{k}\right)
$$

Since we have

$$
-1=\xi_{0}<\xi_{1}<\cdots<\xi_{N-1}<\xi_{N}=1,
$$

we infer that

$$
0=\eta_{N}<\eta_{N-1}<\cdots<\eta_{1}<\eta_{0}=\pi
$$

REMARK 1.1. Since $L_{N}$ is even (resp. odd) when $N$ is even (resp. odd), we see that

$$
\xi_{N-k}=-\xi_{k}, \quad \text { for } 1 \leq k \leq N-1
$$

The matrices $M_{S}$ and $M_{F}$ are diagonal; we define the diagonal elements of $M_{F}^{-1} M_{S}$ as:

$$
\sigma_{k}=\frac{2 \rho_{k}}{\xi_{k+1}-\xi_{k-1}}, \quad \text { for } 1 \leq k \leq N-1
$$

We make the convention that $\sigma_{0}=\sigma_{N}=0$.

REMARK 1.2. It has been proved in Lemma 3.1. of [17] and in Lemma 2.1. of [4] that $\sigma_{k}$ is bounded independently of $k$ and $N$. Precise estimates of $\sigma_{k}$ are available in Parter's Theorem 3.1. of [14]. This result is of great importance since the quantities $\sigma_{k}$ appear in several problems related with spectral methods [5, 4].

Define the discrete $H^{1}$ norm by

$$
\|U\|_{\mathbb{H}_{N}^{1}}=\left(U^{*} K_{F} U\right)^{1 / 2}=\left(\sum_{k=0}^{N-1} \frac{\left|U_{k+1}-U_{k}\right|^{2}}{\xi_{k+1}-\xi_{k}}\right)^{1 / 2} ;
$$

the equivalence between $M_{S}^{1 / 2} M_{F}^{-1 / 2} K_{F} M_{F}^{-1 / 2} M_{S}^{1 / 2}$ and $K_{F}$ is equivalent to the existence of a constant $C>0$ independent of $N$ such that

$$
C^{-1}\|U\|_{\mathbb{H}_{N}^{1}} \leq\left\|M_{F}^{-1 / 2} M_{S}^{1 / 2} U\right\|_{\mathbb{H}_{N}^{1}} \leq C\|U\|_{\mathbb{H}_{N}^{1}}
$$

Here, as is classical, we had to extend the definition of $U_{k}$ by letting $U_{0}=U_{N}=0$. 
So, let us consider the square of the $\mathbb{H}_{N}^{1}$ norm of $M_{F}^{-1 / 2} M_{S}^{1 / 2} U$ given by

$$
\sum_{k=0}^{N-1} \frac{\left|\sqrt{\sigma_{k+1}} U_{k+1}-\sqrt{\sigma_{k}} U_{k}\right|^{2}}{\xi_{k+1}-\xi_{k}}
$$

We first decompose $\sqrt{\sigma_{k+1}} U_{k+1}-\sqrt{\sigma_{k}} U_{k}$ as

$$
\frac{\sqrt{\sigma_{k+1}}+\sqrt{\sigma_{k}}}{2}\left(U_{k+1}-U_{k}\right)+\frac{\sqrt{\sigma_{k+1}}-\sqrt{\sigma_{k}}}{2}\left(U_{k+1}+U_{k}\right) .
$$

The contribution of the first term of (1.7) in the estimate of the discrete $H^{1}$ norm is

$$
\max _{k}\left|\frac{\sqrt{\sigma_{k+1}}+\sqrt{\sigma_{k}}}{2}\right|\left(\sum_{k=0}^{N-1} \frac{\left(U_{k+1}-U_{k}\right)^{2}}{\xi_{k+1}-\xi_{k}}\right)^{1 / 2}=\max _{k}\left|\frac{\sqrt{\sigma_{k+1}}+\sqrt{\sigma_{k}}}{2}\right|\|U\|_{\mathbb{H}_{N}^{1}},
$$

and we use Remark 1.2 to conclude.

The main result of [19] consists in proving that the contribution of the second term of (1.7) can also be estimated in terms of the discrete $H^{1}$ norm of $U$. This result can also be deduced from Parter's article [15]. In a first step, we observe that discrete Hölder continuity estimates give

$$
\left|U_{k+1}+U_{k}\right|^{2} \leq\left(2-\left|\xi_{k}\right|-\left|\xi_{k+1}\right|\right)\|U\|_{\mathbb{H}_{N}^{1}}^{2} .
$$

Thus, we are reduced to estimate

$$
\sum_{k=0}^{N-1} \frac{2-\left|\xi_{k}\right|-\left|\xi_{k+1}\right|}{\xi_{k+1}-\xi_{k}}\left|\sqrt{\sigma_{k+1}}-\sqrt{\sigma_{k}}\right|^{2} .
$$

But $\sigma_{k}$ is bounded from above and from below independently of $k$, see Remark 1.2 ; we define

$$
\mu_{k}=\frac{2-\left|\xi_{k}\right|-\left|\xi_{k+1}\right|}{\xi_{k+1}-\xi_{k}}\left|\frac{1}{\sigma_{k+1}}-\frac{1}{\sigma_{k}}\right|^{2}
$$

which is algebraically simpler but analytically equivalent to the expression appearing in (1.8); according to Lemma 5.7., page 106 of [19], it suffices to show

$$
\Sigma_{N}=\sum_{k=0}^{N-1} \mu_{k} \text { is bounded independently of } N .
$$

Henceforth, we make the convention $1 / \sigma_{0}=1 / \sigma_{N}=0$.

We deduce from symmetry (1.5), formulas (1.3), (1.6) and (1.9) that

$$
\mu_{N-k}=\mu_{k-1}, \quad 1 \leq k \leq N .
$$

Denote by $\lfloor r\rfloor$ the largest integer at most equal to the real $r$. Define $N^{\prime}=\left\lfloor\frac{N-1}{2}\right\rfloor$; it suffices to estimate

$$
\Sigma_{N}^{\prime}=\sum_{k=0}^{N^{\prime}} \mu_{k}
$$


since $\Sigma_{N} \leq 2 \Sigma_{N}^{\prime}$.

Therefore from the definitions (1.9), (1.6) and (1.3) of $\mu_{k}, \sigma_{k}$ and $\rho_{k}$, we have to provide asymptotic expansions for $L_{N}$ and for the zeroes $\xi_{k}$ of $L_{N}^{\prime}$; we start from classical integral or asymptotic formulas for Jacobi polynomials that can be found in the literature.

For the reader's convenience, it is advisable to consult the fourth edition of Szegö's book [20], which is the most complete.

We partition the interval $\left\{0, \cdots, N^{\prime}\right\}$ into three subintervals:

$$
\begin{aligned}
& \{0, \cdots, K\}, \\
& \{K+1, \cdots,\lfloor\Lambda N\rfloor-1\} \text { and } \\
& \left\{\lfloor\Lambda N\rfloor, \cdots, N^{\prime}\right\},
\end{aligned}
$$

where $K$ is bounded and will be chosen later, and $\Lambda$ belongs to the open interval $(0,1 / 2)$.

Let us begin with the leftmost region $0 \leq k \leq K$, where, since $K$ is kept finite, it suffices to find the limit of $\mu_{k}$ for $N$ tending to infinity. Asymptotics for the Legendre polynomials and their derivatives in this region are available as follows: if $N$ tends to infinity and $z$ is bounded by $\pi K$, then

$$
L_{N}\left(\cos \frac{z}{N}\right) \sim J_{0}(z)
$$

where $J_{0}$ is the classical Bessel function; an analogous statement holds for $L_{N}^{\prime}$ (formula (8.1.1) of Szegö [20]). If $z_{k}$ denotes the $k$-th positive zero of the Bessel function $J_{1}$, we find for $k \geq 1[19]$ :

$$
\lim _{N \rightarrow+\infty} \mu_{k}=\frac{z_{k+1}^{2}+z_{k}^{2}}{64\left(z_{k+1}^{2}-z_{k}^{2}\right)}\left|\left(z_{k+1}^{2}-z_{k-1}^{2}\right) J_{0}^{2}\left(z_{k}\right)-\left(z_{k+2}^{2}-z_{k}^{2}\right) J_{0}^{2}\left(z_{k+1}\right)\right|^{2} .
$$

The estimate needed for Theorem 5.8., page 108 of [19] is a direct consequence of the above statement. We do not treat the region $0 \leq k \leq K$ in this article, since we do not need new asymptotics.

Another result from Szegö's book [20], formula (8.21.14), is: if $\Lambda$ belongs to $(0,1 / 2)$ and $\pi \Lambda N \leq z \leq \pi(1-\Lambda) N$, we have

$$
\begin{aligned}
& P_{N}^{(1 / 2)}(\cos (z / N))=L_{N}\left(\cos \frac{z}{N}\right) \\
& =2 \omega_{N, 1 / 2} \sum_{\nu=0}^{p-1} \omega_{\nu, 1 / 2} \frac{1 \times 3 \cdots \times(2 \nu-1)}{(2 N-1)(2 N-3) \cdots(2 N-2 \nu+1)} \\
& \quad \times \frac{\cos ((N-\nu+1 / 2) z / N-(\nu+1 / 2) \pi / 2)}{(2 \sin (z / N))^{\nu+1 / 2}}+O\left(N^{-p-1 / 2}\right)
\end{aligned}
$$

where $\omega_{N, 1 / 2}$ is an explicitly known number and the remainder is uniform over the interval $[\pi \Lambda N, \pi(1-\Lambda) N]$. We also have analogous uniform asymptotics for $L_{N}^{\prime}$, $L_{N}^{\prime \prime}$ and $L_{N}^{\prime \prime \prime}$; therefore, in the rightmost region $\lfloor\Lambda N\rfloor \leq k \leq N^{\prime}$ and thanks to a quantitative implicit function theorem, we can find an expansion in terms of $k$ and $N$ of the zero $\eta_{k}$ of $\theta \mapsto L_{N}^{\prime}(\cos \theta)$ which lies in a neighborhood of size $O\left(N^{-2}\right)$ about

$$
\eta_{0, k}=\pi-\frac{\pi / 4+k \pi}{N+1 / 2}=\frac{(N-k) \pi+\pi / 4}{N+1 / 2} ;
$$


this result will be proved here as Theorem 2.1 and will lead to an estimate of the quantities $\sigma_{k},\lfloor\Lambda N\rfloor \leq k \leq N^{\prime}$ in Corollary 2.4.

There remains to treat the intermediate region, i.e. $z$ between $\pi K$ and $\pi \Lambda N$; it corresponds to $K \leq k \leq\lfloor\Lambda N\rfloor$. This case is not treated in the literature, and we had to devise the estimates and their proof, using the stationary phase method.

Denote by $P_{N}^{(\lambda)}$ the ultra-spherical polynomial of degree $N$ over the interval $[-1,1]$, i.e. the orthogonal polynomial of degree $N$ relatively to the weight $\left(1-x^{2}\right)^{\lambda-1 / 2}$.

REMARK 1.3. The Legendre polynomial $L_{N}$ of degree $N$ is precisely equal to $P_{N}^{(1 / 2)}$, and as a consequence of (4.7.14) from [20], $L_{N}^{\prime}$ is equal to $P_{N-1}^{(3 / 2)}$ and $L_{N}^{\prime \prime}$ is equal to $P_{N-2}^{(5 / 2)}$ up to multiplicative constants.

In order to find asymptotics in the intermediate region, we write an integral representation for $P_{N}^{(\lambda)}$ :

$$
P_{N}^{(\lambda)}(x)=\frac{2^{1-2 \lambda}}{(\Gamma(\lambda))^{2}} \frac{\Gamma(N+2 \lambda)}{N !} \int_{0}^{\pi}\left(x+i \sqrt{1-x^{2}} \cos \varphi\right)^{N} \sin ^{2 \lambda-1} \varphi d \varphi .
$$

We apply the principle of the stationary phase method as described in Lemma 7.7.3 of Hörmander's book [12], but we cannot apply directly the lemma, since the phase is not equal to a large parameter multiplied by a real function of all the other variables: it is a complex function of the large parameter $N$ and all the other variables. We set

$$
\chi_{N}=-i N \sin (z / N) e^{-i z / N}
$$

and, for $\lambda$ such that $2 \lambda-1$ is an even integer, we eventually find polynomials $Q_{\nu, \lambda}$ such that

$$
\begin{aligned}
& \mid P_{N}^{(\lambda)}(\cos (z / N)) \\
& -2 \sqrt{\pi} \frac{2^{1-2 \lambda}}{\Gamma(\lambda)^{2}} \frac{\Gamma(N+2 \lambda)}{N !} \operatorname{Re}\left\{i e^{i z} \sum_{\nu=\lambda-1 / 2}^{\ell-1} \chi_{N}^{-(\nu+1 / 2)} Q_{\nu, \lambda}\left(\chi_{N} / N\right)\right\} \mid \\
& \quad \leq C(K, \Lambda, \ell, \lambda)\left(N^{-1}+z^{-1}\right)^{\ell-2 \lambda+1} ;
\end{aligned}
$$

here $\chi_{N}^{-(\nu+1 / 2)}$ is the principal determination and $C(K, \Lambda, \ell, \lambda)$ depends only on its arguments (Theorem 3.14). Finally, we use once again a quantitative implicit function theorem to obtain an asymptotic expansion of the zero of $L_{N}^{\prime}$ which lies in a neighborhood of size $O\left(1 / N^{2}\right)$ about $\pi(N-k+1 / 4) /(N+1 / 2)$, for $K \leq k \leq\lfloor\Lambda N\rfloor$ (Corollary 3.17); this asymptotic yields an expansion for $\sigma_{k}, K \leq k \leq\lfloor\Lambda N\rfloor$ at Corollary 3.19. Hence we obtain in [19] an estimate on the sum of the $\mu_{k}$ 's for $K \leq k \leq\lfloor\Lambda N\rfloor$.

The article is organized as follows: in section 2, we compute the asymptotics of the zeroes in the rightmost region thanks to an implicit functions theorem and we expand the ratios $\sigma_{k}$. Section 3, devoted to the intermediate region, is split into four sections: in section 3.1, we explain the proof strategy; in section 3.2, we prove a general lemma of stationary or non stationary phase method and we apply it in section 3.3 to obtain expansions of Legendre polynomials; we finally obtain asymptotics of the zeroes of their derivative and of the quantities $\sigma_{k}$ in section 3.4. 
2. The region $\lfloor\Lambda N\rfloor \leq k \leq N^{\prime}$. In order to obtain asymptotics for $\mu_{k}$ in the index range $k \in\left\{\lfloor\Lambda N\rfloor, \cdots, N^{\prime}\right\}$ in [19] as explained in the introduction, we first need asymptotics for the zeroes of $P_{N}^{(3 / 2)}=L_{N+1}^{\prime}$.

It is more convenient to state the following theorem in an interval which is symmetric about $N / 2$ :

Theorem 2.1. Define

$$
\theta_{0, k}=\frac{\pi / 4+k \pi}{N+3 / 2}
$$

Then for all $\Lambda \in(0,1 / 2)$, there exist $C, C^{\prime}$ such that for all $N \geq 2$ and for all integer $k$ in $\{\lfloor\Lambda N\rfloor, \cdots,\lceil(1-\Lambda) N\rceil\}$, there exists a unique zero $\theta_{k}$ of $P_{N}^{(3 / 2)}(\cos \theta)$ in a ball of radius $C^{\prime} / N^{2}$ about $\theta_{0, k}$; moreover the following estimate holds

$$
\left|\theta_{k}-\theta_{0, k}+\frac{3}{8 N^{2} \tan \theta_{0, k}}-\frac{9}{8 N^{3} \tan \theta_{0, k}}\right| \leq C N^{-4} .
$$

Proof. The idea of the proof is to use the quantitative implicit function theorem given in [6]; let us state it here for the reader's convenience:

Lemma 2.2. Let $X$ and $Z$ be Banach spaces, and let $f$ be a $C^{2}$ function from a neighborhood $\mathcal{U}$ of $x_{0} \in X$ to $Z$. Let $z_{0}=f\left(x_{0}\right)$. Assume that $A=D f\left(x_{0}\right)$ has a bounded inverse $A^{-1}$. Assume that the ball of radius $\rho$ and of center $x_{0}$ is included in $\mathcal{U}$. Let

$$
M=\sup _{|\xi| \leq \rho}\left\|A^{-1} D^{2} f\left(x_{0}+\xi\right)\right\|
$$

There exist constants $a$ and $K$ given by

$$
a=\min \left(1,(2 \rho M)^{-1}\right), \quad K=\frac{3 a \rho}{4}
$$

such that if $\left|A^{-1} z_{0}\right| \leq K$, the equation

$$
f(x)=0
$$

possesses a unique solution in the ball $\left\{\left|x-x_{0}\right| \leq a \rho\right\}$; moreover, this solution satisfies

$$
\left|x-x_{0}\right| \leq 2\left|A^{-1} z_{0}\right| \text { and }\left|x-x_{0}+A^{-1} z_{0}\right| \leq 2 M\left|A^{-1} z_{0}\right|^{2} .
$$

As $P_{N}^{(3 / 2)}$ has the same parity as $N$, the set of zeroes of $P_{N}^{(3 / 2)}$ is invariant by the symmetry $x \mapsto-x$, and therefore, at the index level, $\theta_{k}$ is a zero of $P_{N}^{(3 / 2)}(\cos \theta)$ iff $\theta_{N-k}$ is a zero of $P_{N}^{(3 / 2)}(\cos \theta)$, and moreover, $\theta_{N-k}=\pi-\theta_{k}$. Therefore, it suffices to prove the lemma for $\Lambda N \leq k \leq N^{\prime}$.

The definition of the binomial coefficients is extended for all $x \in \mathbb{C}$ and all integer $l \geq 0$ as

$$
\left(\begin{array}{l}
x \\
l
\end{array}\right)=\frac{x(x-1) \cdots(x-l+1)}{l !}
$$


this expression vanishes if $x$ is set equal to 0 or if $l$ is a negative integer. We use the notation

$$
\omega_{N, \lambda}=\left(\begin{array}{c}
N+\lambda-1 \\
N
\end{array}\right)=\frac{\Gamma(N+\lambda)}{\Gamma(N+1) \Gamma(\lambda)} .
$$

We exploit the asymptotics of $P_{N}^{(\lambda)}$ given as (8.21.14) of [20] for $\lambda=3 / 2,5 / 2$ and $7 / 2$, since we need an estimate of $\partial^{j} f / \partial \theta^{j}$ for $j=0,1,2$, in order to apply Lemma 2.2 . We write the three term formula

$$
\begin{aligned}
& P_{N}^{(3 / 2)}(\cos \theta)=\frac{2 \omega_{N, 3 / 2}}{(2 \sin \theta)^{3 / 2}}\{\cos ((N+3 / 2) \theta-3 \pi / 4) \\
&-\frac{3}{2(2 N+1)} \frac{\cos ((N+1 / 2) \theta-5 \pi / 4)}{2 \sin \theta} \\
&\left.-\frac{15}{8(2 N+1)(2 N-1)} \frac{\cos ((N-1 / 2) \theta-7 \pi / 4)}{(2 \sin \theta)^{2}}\right\}+O\left(N^{-5 / 2}\right)
\end{aligned}
$$

which is uniform in $\theta$ in $[\Lambda / 2, \pi / 2]$ and in $N$; it is then convenient to define

$$
f(\theta, N)=\frac{(2 \sin \theta)^{3 / 2}}{2 \omega_{N, 3 / 2}} P_{N}^{(3 / 2)}(\cos \theta)
$$

since we seek the unique root $\theta_{k}$ of $f$ which belongs to a small neighborhood of $\theta_{0, k}$, we will have to calculate $f\left(\theta_{0, k}, N\right),(\partial f / \partial \theta)\left(\theta_{0, k}, N\right)$ and to estimate $\partial^{2} f / \partial \theta^{2}$ for $\theta$ in $\left[\theta_{0, k}-r N^{-2}, \theta_{0, k}+r N^{-2}\right]$; we will choose $r$ later. We differentiate (2.4) twice, we use formula (4.7.14) from Szegő [20], viz.

$$
\frac{d}{d x} P_{N}^{(\lambda)}(x)=2 \lambda P_{N-1}^{(\lambda+1)}(x)
$$

and we find

$$
\frac{\partial f}{\partial \theta}(\theta, N)=\frac{3}{2} \frac{f(\theta, N)}{\tan \theta}-\frac{3 \sqrt{2}}{\omega_{N, 3 / 2}} \sin ^{5 / 2} \theta P_{N-1}^{(5 / 2)}(\cos \theta),
$$

and

$$
\begin{aligned}
\frac{\partial^{2} f}{\partial \theta^{2}}(\theta, N)=\frac{3}{4} & \left(\frac{1}{\tan ^{2} \theta}-2\right) f(\theta, N)-\frac{12 \sqrt{2}}{\omega_{N, 3 / 2}} \cos \theta \sin ^{3 / 2} \theta P_{N-1}^{(5 / 2)}(\cos \theta) \\
& +\frac{15 \sqrt{2}}{\omega_{N, 3 / 2}} \sin ^{7 / 2} \theta P_{N-2}^{(7 / 2)}(\cos \theta) .
\end{aligned}
$$

We first calculate $f\left(\theta_{0, k}, N\right)$ with the help of formula (2.3) and we find

$$
\begin{aligned}
& f\left(\theta_{0, k}, N\right)= \\
& (-1)^{k}\left\{\frac{3}{4(2 N+1) \tan \theta_{0, k}}-\frac{15}{16(2 N+1)(2 N-1) \tan \theta_{0, k}}\right\}+O\left(N^{-3}\right) .
\end{aligned}
$$

We can also evaluate $f(\theta, N)$ for $\left|\theta-\theta_{0, k}\right| \leq r N^{-2}$ : by Taylor expansion,

$$
|\cos ((N+3 / 2) \theta-\pi / 4)| \leq r(N+3 / 2) N^{-2},
$$


and therefore

$$
\left|\theta-\theta_{0, k}\right| \leq r N^{-2} \Longrightarrow|f(\theta)|=(r+1) O\left(N^{-1}\right),
$$

the error term being uniform for $k$ between $\lfloor\Lambda N\rfloor$ and $N^{\prime}$.

We calculate now $\partial f / \partial \theta$ at $\left(\theta_{0, k}, N\right)$ : first we substitute the value found at (2.7) into the first term on the right hand side of (2.5); as $\theta_{0, k}$ is bounded away from 0 and $\pi$, this first term is an $O\left(N^{-1}\right)$, uniformly for $\Lambda N \leq k \leq N^{\prime}$. For the second term of the right hand side of (2.5), we need a two-term expansion of $P_{N}^{(5 / 2)}$, namely

$$
\begin{aligned}
P_{N}^{(5 / 2)}(\cos \theta)= & \frac{2 \omega_{N, 5 / 2}}{(2 \sin \theta)^{5 / 2}}(\cos ((N+5 / 2) \theta-5 \pi / 4) \\
& \left.-\frac{15}{8(N+3 / 2)} \frac{\cos ((N+3 / 2) \theta-7 \pi / 4)}{\sin \theta}\right)+O\left(N^{-1 / 2}\right) .
\end{aligned}
$$

The error term is uniform on the interval $[\Lambda / 2, \pi / 2]$.

We replace $N$ by $N-1$ in (2.9) and we observe that

$$
\frac{6 \sqrt{2}(\sin \theta)^{5 / 2} \omega_{N-1,5 / 2}}{(2 \sin \theta)^{5 / 2} \omega_{N, 3 / 2}}=\frac{3}{2} \frac{\omega_{N-1,5 / 2}}{\omega_{N, 3 / 2}}=N,
$$

according to the definition (2.2) of $\omega_{N, \lambda}$. Furthermore,

$$
\cos \left((N+3 / 2) \theta_{0, k}-5 \pi / 4\right)=(-1)^{k-1}
$$

and

$$
\cos \left((N+1 / 2) \theta_{0, k}-7 \pi / 4\right)=(-1)^{k} \sin \left(\theta_{0, k}\right) .
$$

Thus we find the asymptotic

$$
A(k, N)=\frac{\partial f}{\partial \theta}\left(\theta_{0, k}, N\right)=(-1)^{k}(N+15 / 8)+O\left(N^{-1}\right) .
$$

Now, we choose $r$ :

$$
r=\frac{4}{3} \sup \left\{N^{2}\left|f\left(\theta_{0, k}, N\right) / A(k, N)\right|: N \geq 1, \Lambda N \leq k \leq N^{\prime}\right\} ;
$$

our estimates show that indeed $r$ is bounded.

There remains to give an estimate of $\partial^{2} f / \partial \theta^{2}$ over the interval $\left[\theta_{0, k}-r N^{-2}, \theta_{0, k}+\right.$ $\left.r N^{-2}\right]$. The first term in the right hand side of (2.6) is an $O(1 / N)$, thanks to $(2.8)$; the second term in the right hand side of (2.6) is an $O(N)$ in virtue of (2.10) and the expansion (2.9); the last term in the right hand side of (2.6) is estimated with the help of the one-term expansion of $P_{N}^{(7 / 2)}$ given by

$$
P_{N}^{(7 / 2)}(\cos \theta)=\frac{2 \omega_{N, 7 / 2}}{(2 \sin \theta)^{7 / 2}} \cos ((N+7 / 2) \theta-7 \pi / 4)+O\left(N^{3 / 2}\right)
$$

but $\omega_{N-2,7 / 2} / \omega_{N, 3 / 2}=O\left(N^{2}\right)$ and by a Taylor expansion, $\cos ((N+3 / 2) \theta-7 \pi / 4)$ is an $O(r / N)$ on the relevant interval. Therefore, we obtain the estimate

$$
\left|\theta-\theta_{0, k}\right| \leq r N^{-2} \Longrightarrow\left|\frac{\partial^{2} f}{\partial \theta^{2}}(\theta, N)\right|=(r+1) O(N)
$$


and once again, the estimate is uniform with respect to $k$ such that $\Lambda N \leq k \leq N^{\prime}$, to $r$, and to $N$.

We have then $M=O(r+1)=O(1)$ and for all large enough $N, 2 r M N^{-2}$ is strictly less than 1 , so that we may take $a=1$ in the statement of Lemma 2.2. But then $K$ is equal to $3 r / 4 N^{2}$, and by definition of $r,\left|f\left(\theta_{0, k}, N\right) / A(k, N)\right| \leq K$, and the conclusion of the lemma applies. Relation (2.1) is simply the translation to our particular problem of the conclusion of Lemma 2.2. $\mathrm{\square}$

REMARK 2.3. We can compare this result with expansion (2.6a) of Parter's article [14]; using Remark 1.3, we expand formula (2.1) with $N-1$ instead of $N$ and we find exactly formula (2.6a) of [14]. The main interest of our formula is that the remainder is independent of $k$ and that $\theta_{k}$ is expanded to the next order.

We can now prove the following corollary, which gives an estimate of the quantities $\sigma_{k}$.

Corollary 2.4. The quantities $\sigma_{k},\lfloor\Lambda N\rfloor \leq k \leq N^{\prime}$ defined at equation (1.6) have the following expansion :

$$
\sigma_{k}=1+\frac{\pi^{2}}{6 N^{2}}+O\left(1 / N^{3}\right)
$$

where the error term is uniform in $N$ and in $k \in\left\{\lfloor\Lambda N\rfloor, \cdots, N^{\prime}\right\}$.

Proof. We consider now the zero $\eta_{k}$ of $\theta \mapsto L_{N}^{\prime}(\cos \theta)$. Let us define

$$
\eta_{0, k}=\frac{(N-k+1 / 4) \pi}{N+1 / 2}
$$

using Remark 1.3 and equation (2.1) of Theorem 2.1, we have the asymptotic

$$
\eta_{k}=\eta_{0, k}-\frac{3}{8 N^{2} \tan \eta_{0, k}}\left(1-\frac{1}{N}\right)+O\left(N^{-4}\right),
$$

the error term being uniform in $N$ and in $k \in\left\{\lfloor\Lambda N\rfloor, \cdots, N^{\prime}\right\}$.

Let us now compute an expansion of $L_{N}\left(\cos \eta_{k}\right)$, in order to calculate $\rho_{k}$ defined at equation (1.3).

The three term asymptotic expansion of $L_{N}=P_{N}^{(1 / 2)}$ given at equation (1.11) is

$$
\begin{aligned}
& P_{N}^{(1 / 2)}(\cos \theta)=\frac{2 \omega_{N, 1 / 2}}{\sqrt{2 \sin \theta}}\left(T_{1}+T_{2}+T_{3}\right)+O\left(N^{-7 / 2}\right), \\
& \text { where } T_{1}=\cos ((N+1 / 2) \theta-\pi / 4) \text {, } \\
& T_{2}=\frac{1}{2(2 N-1)} \frac{\cos ((N-1 / 2) \theta-3 \pi / 4)}{2 \sin \theta} \text { and } \\
& T_{3}=\frac{9}{8(2 N-1)(2 N-3)} \frac{\cos ((N-3 / 2) \theta-5 \pi / 4)}{(2 \sin \theta)^{2}} .
\end{aligned}
$$

In this subsection, we write for simplicity

$$
t=\tan \left(\eta_{0, k}\right)
$$


We infer from the asymptotic (2.13) the following asymptotics for each of the terms $T_{1}, T_{2}$ and $T_{3}$ when $\theta=\eta_{k}$ :

$$
\begin{aligned}
& T_{1}=(-1)^{N-k}\left(1-\frac{9}{128 N^{2} t^{2}}\right)+O\left(N^{-3}\right), \\
& T_{2}=(-1)^{N-k-1} \frac{1}{8 N}\left(1+\frac{1}{2 N}+\frac{3}{8 N t^{2}}\right)+O\left(N^{-3}\right), \\
& T_{3}=(-1)^{N-k-1} \frac{9}{128 N^{2}}\left(\frac{1}{t^{2}}-1\right)+O\left(N^{-3}\right) .
\end{aligned}
$$

Therefore, the sum $T_{1}+T_{2}+T_{3}$ is

$$
T_{1}+T_{2}+T_{3}=(-1)^{N-k}\left(1-\frac{1}{8 N}+\frac{1}{128 N^{2}}-\frac{3}{16 N^{2} t^{2}}\right)+O\left(N^{-3}\right) .
$$

We also need an expansion for $1 / \sqrt{\sin \eta_{k}}$ : from the Taylor expansion

$$
\sin \eta_{k}=\sin \eta_{0, k}\left(1-\frac{3}{8 N^{2} t^{2}}+O\left(N^{-3}\right)\right)
$$

we infer

$$
\frac{1}{\sqrt{\sin \eta_{k}}}=\frac{1}{\sqrt{\sin \eta_{0, k}}}\left(1+\frac{3}{16 N^{2} t^{2}}+O\left(N^{-3}\right)\right) .
$$

Finally, we get an expansion of $\omega_{N, 1 / 2}$ with the help of Stirling's formula:

$$
\omega_{N, 1 / 2}=\frac{1}{\sqrt{\pi N}}\left(1-\frac{1}{8 N}+\frac{1}{128 N^{2}}+O\left(N^{-3}\right)\right) .
$$

We perform the product of (2.14), (2.16) and (2.17), and we find

$$
L_{N}\left(\cos \eta_{k}\right)=(-1)^{N-k} \frac{\sqrt{2}}{\sqrt{\pi N \sin \eta_{0, k}}}\left(1-\frac{1}{4 N}+\frac{1}{32 N^{2}}+O\left(N^{-3}\right)\right) .
$$

Observe that the error term in (2.18) is uniform in $N$ and in $k \in\left\{\lfloor\Lambda N\rfloor, \cdots, N^{\prime}\right\}$.

In order to calculate $\sigma_{k}$, we need an asymptotic of $\xi_{k+1}-\xi_{k-1}$ : we write a Taylor expansion of $\xi_{k \pm 1}=\cos \eta_{k \pm 1}$ at $\eta_{k}$, and we obtain

$$
\begin{aligned}
& \xi_{k+1}-\xi_{k-1}= \\
& \quad \sin \eta_{k}\left(\eta_{k-1}-\eta_{k+1}\right)-\frac{1}{2}\left(\left(\eta_{k+1}-\eta_{k}\right)^{2}-\left(\eta_{k-1}-\eta_{k}\right)^{2}\right) \cos \eta_{k} \\
& +\frac{1}{6}\left(\left(\eta_{k+1}-\eta_{k}\right)^{3}-\left(\eta_{k-1}-\eta_{k}\right)^{3}\right) \sin \eta_{k}+O\left(N^{-4}\right) .
\end{aligned}
$$

Another Taylor expansion gives $\eta_{k \pm 1}-\eta_{k}$ :

$$
\eta_{k \pm 1}-\eta_{k}=\left(\eta_{0, k \pm 1}-\eta_{0, k}\right)\left(1+\frac{3\left(1+1 / t^{2}\right)}{8 N^{2}}\right)+O\left(N^{-4}\right) .
$$

Therefore, we obtain with the help of (2.15):

$$
\frac{\xi_{k+1}-\xi_{k-1}}{2}=\sin \eta_{0, k} \frac{\pi}{N+1 / 2}\left(1+\frac{3}{8 N^{2}}-\frac{\pi^{2}}{6 N^{2}}\right)+O\left(N^{-4}\right) .
$$


We put together (2.18) and (2.19) and we obtain the expansion of $\sigma_{k}$, given by

$$
\sigma_{k}=1+\frac{\pi^{2}}{6 N^{2}}+O\left(1 / N^{3}\right)
$$

3. The region $K \leq k \leq\lfloor\Lambda N\rfloor$. Let us find the asymptotics of the zeroes of the derivatives of Legendre polynomials in the intermediate region, which is the most difficult to handle.

The goal of this section is to infer Corollary 3.17 from a long chain of results; we state it here, for the reader to understand our final aim; it will be stated again at its natural place.

Corollary 3.17 1. Define

$$
\theta_{0, k}=\frac{\pi(N-k+1 / 4)}{N+1 / 2} .
$$

Then for all $K>0$ and for all $\Lambda \in(0,1 / 2)$, there exist $C, C^{\prime}$ such that for all $N \geq 2$ and for all integer $k$ in $\{K, \cdots,\lfloor\Lambda N\rfloor\}$, there exists a unique zero $\theta_{k}$ of $L_{N}^{\prime}(\cos \theta)$ in a ball of radius $C^{\prime} / N^{2}$ about $\theta_{0, k}$; moreover the following estimate holds

$$
\left|\theta_{k}-\theta_{0, k}-\frac{13}{8 N^{2} \tan \theta_{0, k}}+\frac{49}{12 N^{3} \tan \theta_{0, k}}\right| \leq C\left(\left(N^{-1}+K^{-1}\right)^{4}\right) .
$$

For that purpose, we first calculate expansions of Legendre polynomials and we begin by explaining the strategy of the proof.

3.1. The strategy of the proof. In order to calculate formulas for $L_{N}^{\prime}(\cos \theta)$ and for $L_{N}(\cos \theta)$ and their derivatives, we will use the integral representation given by formula (4.10.3) of Szegö [20]: define

$$
Z(\lambda, N)=\frac{2^{1-2 \lambda}}{(\Gamma(\lambda))^{2}} \frac{\Gamma(N+2 \lambda)}{N !},
$$

the following formula holds for $\lambda>0$ and all $x \in[-1,1]$ :

$$
P_{N}^{(\lambda)}(x)=Z(\lambda, N) \int_{0}^{\pi}\left(x+i \sqrt{1-x^{2}} \cos \varphi\right)^{N} \sin ^{2 \lambda-1} \varphi d \varphi .
$$

In fact, this formula is also true for all $x \in \mathbb{C}$, provided that we choose the appropriate determination of the square root appearing in the integrand.

We define the two following functions:

$$
f_{N}(z, \varphi)=(\cos (z / N)+i \sin (z / N) \cos \varphi)^{N}
$$

and $g_{N}$ such that $f_{N}=\exp g_{N}$, i.e.

$$
g_{N}(z, \varphi)=N \ln (\cos (z / N)+i \sin (z / N) \cos \varphi)
$$

where we have taken the principal determination of the logarithm. 
We infer from (3.2) the expression of the ultra-spherical polynomials at $x=$ $\cos (z / N)$ :

$$
\begin{aligned}
P_{N}^{(\lambda)}(\cos (z / N)) & =Z(\lambda, N) \operatorname{Re}\left(\int_{0}^{\pi} f_{N}(z, \varphi) \sin ^{2 \lambda-1} \varphi d \varphi\right) \\
& =Z(\lambda, N) \operatorname{Re}\left(\int_{0}^{\pi} \exp g_{N}(z, \varphi) \sin ^{2 \lambda-1} \varphi d \varphi\right)
\end{aligned}
$$

In our calculations, we will often need the following useful remark:

REMARK 3.1. The function $g_{N}$ is an even function of $\varphi$ and therefore its derivatives of odd order will vanish at $\varphi=0$.

We shall seek an asymptotic formula for $\int_{0}^{\pi} f_{N}(z, \varphi) \sin ^{2 \lambda-1} \varphi d \varphi$.

Let $\delta$ belong to $[0, \pi / 4[$ and $\psi$ be a cut-off function having the following properties

$$
\begin{gathered}
\psi \text { is even, } \pi \text {-periodic, of class } C^{\infty} \text { with values in }[0,1], \\
\psi \text { is equal to } 1 \text { over }[0, \delta] \text { and to } 0 \text { over }[2 \delta, \pi / 2] .
\end{gathered}
$$

The function $\psi$ will enable us to localize difficulties.

Therefore, we can write

$$
\begin{aligned}
\int_{0}^{\pi} f_{N}(z, \varphi) \sin ^{2 \lambda-1} \varphi d \varphi= & \int_{0}^{\pi} \psi(\varphi) f_{N}(z, \varphi) \sin ^{2 \lambda-1} \varphi d \varphi \\
& +\int_{0}^{\pi}(1-\psi(\varphi)) f_{N}(z, \varphi) \sin ^{2 \lambda-1} \varphi d \varphi
\end{aligned}
$$

We will apply a stationary phase strategy, meaning that the second integral in the right hand side of (3.5) is small: this statement is made precise at Corollary 3.6. The main effort is devoted to the estimate of

$$
\begin{aligned}
& \int_{0}^{\pi} \psi(\varphi) f_{N}(z, \varphi) \sin ^{2 \lambda-1} \varphi d \varphi=\int_{0}^{\pi} \psi(\varphi) \exp g_{N}(z, \varphi) \sin ^{2 \lambda-1} \varphi d \varphi \\
& =2 \operatorname{Re}\left(\int_{0}^{\pi / 2} \psi(\varphi) \exp g_{N}(z, \varphi) \sin ^{2 \lambda-1} \varphi d \varphi\right)
\end{aligned}
$$

by the stationary phase method.

We use a homotopy technique as in Hörmander's proof. Let $q_{N}$ be the quadratic part of Taylor's expansion of $g_{N}(z, \cdot)$ at 0 , i.e.

$$
\begin{aligned}
q_{N}(z, \varphi) & =g_{N}(z, 0)+\frac{\varphi^{2}}{2} \frac{\partial^{2} g_{N}}{\partial \varphi^{2}}(z, 0) \\
& =i z-\frac{i N \varphi^{2}}{2} \sin (z / N) e^{-i z / N}
\end{aligned}
$$

and define

$$
R_{N}(z, \varphi)=g_{N}(z, \varphi)-q_{N}(z, \varphi) .
$$

The extensions of $g_{N}$ and $f_{N}$ as functions over $\mathbb{R} \times[0, \pi] \times[0,1]$ are given by

$$
g_{N}(z, \varphi, s)=s g_{N}(z, \varphi)+(1-s) q_{N}(z, \varphi)=q_{N}(z, \varphi)+s R_{N}(z, \varphi)
$$


and

$$
f_{N}(z, \varphi, s)=\exp g_{N}(z, \varphi, s) .
$$

The double of the real part of the integral

$$
\mathcal{I}_{N, \lambda}(z, s)=\int_{0}^{\pi / 2} \psi(\varphi) \exp g_{N}(z, \varphi, s) \sin ^{2 \lambda-1} \varphi d \varphi
$$

is equal to (3.6) for $s=1$ and for $s=0$, it can be expanded simply. Therefore, in order to estimate $\mathcal{I}_{N, \lambda}(z, 1)$, we use a Taylor expansion at $s=0$, viz.

$$
\left|\mathcal{I}_{N, \lambda}(z, 1)-\sum_{l=0}^{k-1} \frac{1}{l !} \frac{\partial^{l} \mathcal{I}_{N, \lambda}}{\partial s^{l}}(z, 0)\right| \leq \max _{0 \leq s \leq 1}\left|\frac{1}{k !} \frac{\partial^{k} \mathcal{I}_{N, \lambda}}{\partial s^{k}}(z, s)\right| .
$$

We produce explicit approximations of the terms $\left(\partial^{l} \mathcal{I}_{N, \lambda} / \partial s^{l}\right)(z, 0)$ and the formula for $\mathcal{I}_{N, \lambda}(z, 1)$ will be a sum of these explicit approximations plus a sum of remainders which have to be estimated. There are two kinds of remainders : one comes from the difference between $\left(\partial^{l} \mathcal{I}_{N, \lambda} / \partial s^{l}\right)(z, 0)$ and its approximation, and another one comes from the right hand side of $(3.12)$.

The derivative $\partial^{l} \mathcal{I}_{N, \lambda} / \partial s^{l}$ is given by

$$
\frac{\partial^{l} \mathcal{I}_{N, \lambda}}{\partial s^{l}}(z, s)=\int_{0}^{\pi / 2} \psi(\varphi) R_{N}^{l}(z, \varphi) \exp g_{N}(z, \varphi, s) \sin ^{2 \lambda-1} \varphi d \varphi .
$$

In order to obtain the explicit approximations of $\left(\partial^{l} \mathcal{I}_{N, \lambda} / \partial s^{l}\right)(z, 0)$ mentioned above, we first approximate $R_{N}$ by its Taylor expansion. Let $r_{N}$ be the Taylor expansion of $R_{N}(z, \cdot)$ with respect to $\varphi$ of order $2(k+1)$ at 0 :

$$
r_{N}(z, \varphi)=\sum_{\gamma=2}^{k+1} \frac{\varphi^{2 \gamma}}{(2 \gamma) !} \frac{\partial^{2 \gamma} g_{N}}{\partial \varphi^{2 \gamma}}(z, 0)
$$

observe here that we do not have odd powers of $\varphi$, since $R_{N}$ is even. Corollary 3.11 gives an estimate of

$$
\int_{0}^{\pi / 2} \psi(\varphi)\left(R_{N}^{l}(z, \varphi)-r_{N}^{l}(z, \varphi)\right) \exp q_{N}(z, \varphi) \sin ^{2 \lambda-1} \varphi d \varphi
$$

Then, the usable explicit approximations will be calculated using Lemma 7.7.3 of Hörmander [12] for the following integrals :

$$
\int_{0}^{\pi / 2} \psi(\varphi) r_{N}^{l}(z, \varphi) \exp g_{N}(z, \varphi, s) \sin ^{2 \lambda-1} \varphi d \varphi
$$

which is done at Corollary 3.13 .

Finally, we estimate $\partial^{k} \mathcal{I}_{N, \lambda} / \partial s^{k}$, which is the main part of the right hand side of equation (3.12), at Corollary 3.9.

The usable algebraic expressions of the terms which appear in asymptotics of $P_{N}^{(\lambda)}$ are given first in general form at Theorem 3.14 for $2 \lambda-1$ an even integer, and explicit results for $\lambda=1 / 2,3 / 2,5 / 2$ and $7 / 2$ are given at Corollary 3.15 . 
3.2. A general lemma of stationary and non-stationary methods. We show a general lemma to help proving all the estimates explained in section 3.1.

We need several preliminary technical results. First we estimate $\exp \left(g_{N}(z, \varphi, s)\right)$.

LEMmA 3.2. For all $N \geq 2$, for all $\varphi \in[0, \pi]$, for all $z \in \mathbb{R}^{+}$and for all $s \in[0,1]$,

$$
\left|\exp \left(g_{N}(z, \varphi, s)\right)\right| \leq 1
$$

Proof. It suffices to check $\operatorname{Re} g_{N}(z, \varphi, s) \leq 0$ which is true provided that $\operatorname{Re} g_{N}(z, \varphi)$ and $\operatorname{Re} q_{N}(z, \varphi)$ are less than or equal to 0 .

The real part of $g_{N}$ is $N \ln \left(1-\sin ^{2}(z / N) \sin ^{2} \varphi\right) / 2$ which has the required sign. The real part of $q_{N}$ is $-N \varphi^{2} \sin ^{2}(z / N) / 2$ which is also less than or equal to 0 .

Differentiating composite functions can be done with the help of Faa di Bruno's formula, see for instance Lemma II.2.8 of Hairer [10].

For $m \in \mathbb{N}$, let $\mathcal{C}(m)$ be the set of multi-indices $\gamma=\left(\gamma_{1}, \gamma_{2}, \cdots\right) \in \mathbb{N}^{\mathbb{N}^{*}}$ such that $\gamma_{1} \geq \gamma_{2} \geq \cdots$ and such that $\sum_{i \in \mathbb{N}} \gamma_{i}=m$. Therefore $\gamma_{i}$ vanishes beyond a certain rank; we denote by $l(\gamma)$ the largest integer $i$ such that $\gamma_{i} \geq 1$ and we observe that $l(\gamma) \leq m$. For instance, if we only write the non zero terms of each $\gamma, \mathcal{C}(3)$ is equal to $\{(3),(2,1),(1,1,1)\}$.

Faa di Bruno's formula states that there exist integer constants $C(\gamma, m)$ such that

$$
\frac{d^{m}}{d x^{m}} A \circ B=\sum_{\gamma \in \mathcal{C}(m)} C(\gamma, m)\left(A^{(l(\gamma))} \circ B\right) \prod_{j=1}^{l(\gamma)} B^{\left(\gamma_{j}\right)}
$$

Here, $A$ and $B$ are functions of one real variable. In consequence, if we take $A(x)=x^{k}$, with $k \in \mathbb{Z}$, we can calculate for any function $B$ the derivatives of $B^{k}$ :

$$
\frac{d^{m}}{d x^{m}} B^{k}=\sum_{\gamma \in \mathcal{C}(m)} C(\gamma, m)\left(\begin{array}{c}
k \\
l(\gamma)
\end{array}\right) l(\gamma) ! B^{k-l(\gamma)} \prod_{j=1}^{l(\gamma)} B^{\left(\gamma_{j}\right)}
$$

Let us estimate now the derivatives of $\left(\partial g_{N} / \partial \varphi\right)^{-1}$, which will arise later when we will perform several integrations by part, and let us also estimate the derivatives of $g_{N}$.

LEMma 3.3. For all $k \in \mathbb{N}$, for all $\alpha>0$, there exists $C>0$ such that for all $N \geq 2$, for all $\varphi \in(0, \pi-\alpha]$ and for all $z \in[\pi K, \pi \Lambda N]$, the following estimates hold

$$
\left|\frac{\partial^{k}}{\partial \varphi^{k}}\left(\frac{1}{\partial g_{N} / \partial \varphi}\right)(z, \varphi)\right| \leq \frac{C}{\varphi^{k+1}}\left(N^{-1}+z^{-1}\right)
$$

and

$$
\left|\frac{\partial^{k+1} g_{N}}{\partial \varphi^{k+1}}(z, \varphi)\right| \leq C z
$$

Proof. Write

$$
\begin{gathered}
\nu(\varphi)=\sin \varphi(\text { as in numerator) and } \\
d_{N}(z, \varphi)=(\cos (z / N)+i \sin (z / N) \cos \varphi) \text { (as in denominator) }
\end{gathered}
$$


Then the first derivative of $g_{N}$ and its inverse are

$$
\frac{\partial g_{N}}{\partial \varphi}(z, \varphi)=-i N \sin (z / N) \frac{\nu(\varphi)}{d_{N}(z, \varphi)}
$$

and

$$
\frac{1}{\partial g_{N} / \partial \varphi}(z, \varphi)=\frac{i}{N \sin (z / N)} \frac{d_{N}(z, \varphi)}{\nu(\varphi)} .
$$

Leibniz formula gives

$$
\frac{\partial^{k}}{\partial \varphi^{k}}\left(\frac{1}{\partial g_{N} / \partial \varphi}\right)=\frac{i}{N \sin (z / N)} \sum_{m=0}^{k}\left(\begin{array}{c}
k \\
m
\end{array}\right) \frac{\partial^{k-m} d_{N}}{\partial \varphi^{k-m}} \frac{\partial^{m}}{\partial \varphi^{m}}\left(\frac{1}{\nu}\right) .
$$

The successive derivatives of $1 / \nu$ are computed using (3.17) for $k=-1$; up to arithmetic constants, the terms we find in (3.23) are of the form

$$
\frac{i}{N \sin (z / N)} \frac{\partial^{k-m} d_{N}}{\partial \varphi^{k-m}} \nu^{-1-l(\gamma)} \prod_{j=1}^{l(\gamma)} \frac{\partial^{\gamma_{j}} \nu}{\partial \varphi^{\gamma_{j}}}
$$

we substitute the expressions of the derivatives

$$
\frac{\partial^{j} d_{N}}{\partial \varphi^{j}}(z, \varphi)=i \sin (z / N) \cos (\varphi+j \pi / 2), \text { for all } j \geq 1
$$

and

$$
\frac{\partial^{n} \nu}{\partial \varphi^{n}}(\varphi)=\sin (\varphi+n \pi / 2)
$$

into (3.24): for $m=k$, the expressions (3.24) are equal to

$$
\frac{i(\cos (z / N)+i \sin (z / N) \cos \varphi)}{N \sin (z / N)} \frac{1}{\sin ^{1+l(\gamma)} \varphi} \prod_{j=1}^{l(\gamma)} \sin \left(\varphi+\gamma_{j} \pi / 2\right)
$$

which can be estimated by $C /\left(z \varphi^{k+1}\right)$.

For $m \leq k-1$, the terms (3.24) are of the form

$$
-\frac{1}{N} \cos (\varphi+(k-m) \pi / 2) \frac{1}{\sin ^{1+l(\gamma)} \varphi} \prod_{j=1}^{l(\gamma)} \sin \left(\varphi+\gamma_{j} \pi / 2\right)
$$

which can be estimated by $C /\left(N \varphi^{k+1}\right)$, proving thus (3.18).

Similarly, we write a Leibniz formula for $\partial^{k+1} g_{N} / \partial \varphi^{k+1}$ :

$$
\frac{\partial^{k+1} g_{N}}{\partial \varphi^{k+1}}(z, \varphi)=-i N \sin (z / N) \sum_{m=0}^{k}\left(\begin{array}{c}
k \\
m
\end{array}\right) \frac{\partial^{k-m} \nu}{\partial \varphi^{k-m}}(\varphi) \frac{\partial^{m}}{\partial \varphi^{m}}\left(\frac{1}{d_{N}}\right)(z, \varphi) .
$$

We use formula (3.17) with $k=-1$, i.e.

$$
\begin{aligned}
\frac{\partial^{m}}{\partial \varphi^{m}}\left(\frac{1}{d_{N}}\right)(z, \varphi)= & \sum_{\gamma \in \mathcal{C}(m)} C(\gamma, m)(-1)^{l(\gamma)} l(\gamma) ! d_{N}^{-1-l(\gamma)}(z, \varphi) \\
& \times \prod_{j=1}^{l(\gamma)} \frac{\partial^{\gamma_{j}} d_{N}}{\partial \varphi^{\gamma_{j}}}(z, \varphi)
\end{aligned}
$$


up to arithmetic constants, we substitute the values (3.25) and (3.26) of the derivatives of $d_{N}$ and $\nu$ and for $k \geq 1$, Leibniz formula implies that the terms of the sum (3.27) are of the following form

$$
\begin{aligned}
& -i N \sin (z / N) \sin (\varphi+(k-m) \pi / 2) \frac{(i \sin z / N)^{l(\gamma)}}{(\cos z / N+i \sin z / N \cos \varphi)^{1+l(\gamma)}} \\
& \quad \times \prod_{j=1}^{l(\gamma)} \cos \left(\varphi+\gamma_{j} \pi / 2\right) .
\end{aligned}
$$

It is plain that the modulus of (3.29) is at most equal to $N|\sin z / N|$ and the conclusion of the lemma is clear.

The technical lemma 3.5 will be used many times in the foregoing estimates; it depends on the preliminary lemma 3.4.

Let $p \in \mathbb{N}$ and $b \in(0, \pi)$. Let $u$ be a function of class $C^{p}$ over $[\pi K,+\infty) \times[0, b]$; assume that there exist a real $c \geq 2 p$ and a real $l \geq 0$ such that the following norm

$$
\|u\|_{p, c, l}=\max _{0 \leq i \leq p} \max _{N \in \mathbb{N}} \max _{\substack{\varphi \in(0, b] \\ z \in[\pi K, \pi \Lambda N]}} z^{-l} \varphi^{-c+i}\left|\frac{\partial^{i} u}{\partial \varphi^{i}}(z, \varphi)\right|
$$

is finite. We define by induction

$$
\begin{aligned}
U_{0} & =u, \\
U_{m+1} & =\frac{\partial}{\partial \varphi}\left(\frac{U_{m}}{\partial g_{N} / \partial \varphi}\right) \text { for all } m \in\{0, \cdots, p-1\} .
\end{aligned}
$$

We need to estimate the derivatives of the functions (3.31), since they will appear in the integration by parts which will be performed in the stationary and non stationary phase methods.

Lemma 3.4. Let $u$ be a function of class $C^{p}$ over $[\pi K,+\infty) \times[0, b]$; assume that there exist $c \geq 2 p$ and $l \geq 0$ such that $\|u\|_{p, c, l}<+\infty$. Then, there exists $C>0$ such that for all $N \geq 2$, for all $m \in\{0, \cdots, p\}$, for all $q \in\{0, \cdots, p-m\}$, for all $\varphi \in[0, b]$ and for all $z \in[\pi K, \pi \Lambda N]$,

$$
\left|\frac{\partial^{q}}{\partial \varphi^{q}} U_{m}(z, \varphi)\right| \leq C\|u\|_{q+m, c, l}\left(N^{-1}+z^{-1}\right)^{m-l} \varphi^{c-q-2 m} .
$$

Proof. Let us prove this lemma by induction on $m$. We have

$$
\frac{\partial^{q} U_{0}}{\partial \varphi^{q}}(z, \varphi)=\frac{\partial^{q} u}{\partial \varphi^{q}}(z, \varphi)
$$

and thus using the hypothesis made on $\|u\|_{p, c, l}$, we infer that

$$
\left|\frac{\partial^{q} U_{0}}{\partial \varphi^{q}}(z, \varphi)\right| \leq\|u\|_{q, c, l} z^{l} \varphi^{c-q} \leq C\|u\|_{q, c, l}\left(N^{-1}+z^{-1}\right)^{-l} \varphi^{c-q} .
$$

Assuming that estimate (3.32) is proved for $m$, we use definition (3.31) and Leibniz formula: 


$$
\frac{\partial^{q} U_{m+1}}{\partial \varphi^{q}}=\sum_{s=0}^{q+1}\left(\begin{array}{c}
q+1 \\
s
\end{array}\right) \frac{\partial^{q+1-s} U_{m}}{\partial \varphi^{q+1-s}} \frac{\partial^{s}}{\partial \varphi^{s}}\left(\frac{1}{\partial g_{N} / \partial \varphi}\right) .
$$

Using the induction hypothesis and Lemma 3.3,

$$
\begin{aligned}
\mid \frac{\partial^{q+1-s} U_{m}}{\partial \varphi^{q+1-s}}(z, \varphi) & \frac{\partial^{s}}{\partial \varphi^{s}}\left(\frac{1}{\partial g_{N} / \partial \varphi}\right)(z, \varphi) \mid \\
& \leq C\|u\|_{q+m+1-s, c, l}\left(N^{-1}+z^{-1}\right)^{m+1-l} \varphi^{c-q-2-2 m} \\
& \leq C\|u\|_{q+m+1, c, l}\left(N^{-1}+z^{-1}\right)^{m+1-l} \varphi^{c-q-2(m+1)}
\end{aligned}
$$

and the proof of Lemma 3.4 is complete.

Here is our general lemma:

Lemma 3.5. Let $k \in \mathbb{N}^{*}$ and $b \in[0, \pi)$. Take $u$ in $C_{0}^{\infty}([\pi K,+\infty) \times[0, b])$; assume that there exist $l \geq 0$ and $c \geq 2(k+l)$ such that $\|u\|_{k+l, c, l}$ is finite. Then there exists $C$ such that for all $N \geq 2$ and all $z \in[\pi K, \pi \Lambda N]$,

$$
\max _{s \in[0,1]}\left|\int_{0}^{b} u(z, \varphi) \exp g_{N}(z, \varphi, s) d \varphi\right| \leq C\|u\|_{k+l, c, l}\left(N^{-1}+z^{-1}\right)^{k} .
$$

Proof. Thanks to several integrations by part and using definition (3.31), we can write the integral appearing in the left hand side of (3.33) as

$$
\begin{aligned}
& \int_{0}^{b} u(z, \varphi) \exp g_{N}(z, \varphi, s) d \varphi= \\
& \sum_{m=0}^{k+l-1}\left[\frac{(-1)^{m}}{\partial g_{N} / \partial \varphi} U_{m}(z, \varphi) \exp g_{N}(z, \varphi, s)\right]_{0}^{b} \\
& \quad+(-1)^{k+l} \int_{0}^{b} U_{k+l}(z, \varphi) \exp g_{N}(z, \varphi, s) d \varphi
\end{aligned}
$$

Since $u$ is equal to 0 in a neighborhood of $\varphi=b$, for all $m \in\{0, \cdots, k+l-1\}$, for all $z$ in $[\pi K, \pi \Lambda N], U_{m}(z, b)$ vanishes and thus all the integrated terms at $\varphi=b$ disappear:

$$
\begin{aligned}
\int_{0}^{b} u(z, \varphi) \exp g_{N}(z, \varphi, s) d \varphi=\sum_{m=0}^{k+l-1}(-1)^{m} \frac{U_{m}}{\partial g_{N} / \partial \varphi}(z, 0) \exp g_{N}(z, 0, s) \\
+(-1)^{k+l} \int_{0}^{b} U_{k+l}(z, \varphi) \exp g_{N}(z, \varphi, s) d \varphi .
\end{aligned}
$$

Thanks to Lemmas 3.3 and 3.4, we can estimate all these terms.

Lemma 3.3 with $k=0$ and Lemma 3.4 with $q=0$ give for $m$ in $\{0, \cdots, k+l-1\}$, in the neighborhood of $\varphi=0$,

$$
\frac{U_{m}}{\partial g_{N} / \partial \varphi}(z, \varphi)=O(1)\left(N^{-1}+z^{-1}\right)^{m-l+1} \varphi^{c-2 m-1}
$$


where $O(1)$ is bounded independently of $\varphi \in[0, b], z \in[\pi K, \pi \Lambda N], N \geq 2$ and of finite $l$ and $m$. Since $c \geq 2(k+l)>2 m+1$, we obtain $\left[U_{m} /\left(\partial g_{N} / \partial \varphi\right)\right](z, 0)=0$. Moreover, $\exp g_{N}(z, 0, s)=\exp (i z)$ and thus equation (3.35) becomes

$$
\begin{aligned}
\int_{0}^{b} u(z, \varphi) \exp g_{N}(z, \varphi, s) d \varphi \\
\quad=(-1)^{k+l} \int_{0}^{b} U_{k+l}(z, \varphi) \exp g_{N}(z, \varphi, s) d \varphi .
\end{aligned}
$$

Thanks to Lemma 3.2 and Lemma 3.4 with $m=k+l$ and $q=0$, we obtain estimate (3.33).

3.3. Asymptotics of Legendre polynomials. Now that Lemma 3.5 is proved, we can estimate the integrals displayed in section 3.1.

First, a straightforward corollary of Lemma 3.5 shows that the second integral of the right hand side of (3.5) is small.

Corollary 3.6. Let $\psi$ satisfy conditions (3.4). For all positive integer $k$ and for all $\lambda>0$, there exists $C$ such that for all $N \geq 2$ and for all $z$ in $[\pi K, \pi \Lambda N]$, the following estimate holds:

$$
\left|\int_{0}^{\pi}(1-\psi(\varphi)) \exp g_{N}(z, \varphi) \sin ^{2 \lambda-1} \varphi d \varphi\right| \leq C\left(N^{-1}+z^{-1}\right)^{k} .
$$

Proof. We use Lemma 3.5 with $u(z, \varphi)=(1-\psi(\varphi)) \sin ^{2 \lambda-1} \varphi$ and $b=\pi-\delta / 2$. The function $u$ and its derivatives vanish in a neighborhood of $\varphi=b$ and in the neighborhood $[-\delta, \delta]$ of 0 ; if we set $l=0$ and $c=2 k,\|u\|_{k, 2 k, 0}$ is finite. We infer from Lemma 3.5 that

$$
\begin{aligned}
& \left|\int_{0}^{\pi}(1-\psi(\varphi)) \exp g_{N}(z, \varphi, 1) \sin ^{2 \lambda-1} \varphi d \varphi\right| \\
& =\left|\int_{0}^{\pi-\frac{\delta}{2}}(1-\psi(\varphi)) \exp g_{N}(z, \varphi, 1) \sin ^{2 \lambda-1} \varphi d \varphi\right| \\
& \leq C\|u\|_{k, 2 k, 0}\left(N^{-1}+z^{-1}\right)^{k},
\end{aligned}
$$

where $C$ depends only on $k$, which is estimate (3.36).

In order to apply Lemma 3.5 to the remainder defined by equation (3.12), we need to estimate the derivatives of the powers of $R_{N}$, defined at equation (3.8).

Lemma 3.7. For all $k \in \mathbb{N}^{*}$ and $m \in \mathbb{N}$, there exists $C>0$ such that for all $N \geq 2$, for all $z \in[\pi K, \pi \Lambda N]$ and for all $\varphi \in[0, \pi / 2]$,

$$
\left|\frac{\partial^{m} R_{N}^{k}}{\partial \varphi^{m}}(z, \varphi)\right| \leq C z^{k} \min \left(1, \varphi^{4 k-m}\right) .
$$

Proof. For $k=1$ and $m \leq 3$, Taylor's integral formula gives

$$
\frac{\partial^{m} R_{N}(z, \varphi)}{\partial \varphi^{m}}=\int_{0}^{\varphi} \frac{\partial^{4} g_{N}}{\partial \varphi^{4}}\left(z, \varphi^{\prime}\right) \frac{\left(\varphi-\varphi^{\prime}\right)^{3-m}}{(3-m) !} d \varphi^{\prime},
$$


and for $m \geq 4$,

$$
\frac{\partial^{m} R_{N}}{\partial \varphi^{m}}=\frac{\partial^{m} g_{N}}{\partial \varphi^{m}}
$$

We infer immediately from these relations and the parity of $R_{N}$ with respect to $\varphi$ the estimates

$$
\begin{aligned}
& \left|R_{N}(z, \varphi)\right| \leq C \varphi^{4} z \\
& \left|\frac{\partial R_{N}}{\partial \varphi}(z, \varphi)\right| \leq C \varphi^{3} z \\
& \left|\frac{\partial^{2} R_{N}}{\partial \varphi^{2}}(z, \varphi)\right| \leq C \varphi^{2} z \\
& \left|\frac{\partial^{m} R_{N}}{\partial \varphi^{m}}(z, \varphi)\right| \leq C \varphi z \text { for } m \geq 3, m \text { odd, } \\
& \left|\frac{\partial^{m} R_{N}}{\partial \varphi^{m}}(z, \varphi)\right| \leq C z \text { for } m \geq 4, m \text { even. }
\end{aligned}
$$

Using Faa di Bruno's formula (3.17), we obtain

$$
\frac{\partial^{m} R_{N}^{k}}{\partial \varphi^{m}}(z, \varphi)=\sum_{\gamma \in \mathcal{C}(m)} C(\gamma, m)\left(\begin{array}{c}
k \\
l(\gamma)
\end{array}\right) l(\gamma) ! R_{N}^{k-l(\gamma)}(z, \varphi) \prod_{j=1}^{l(\gamma)} \frac{\partial^{\gamma_{j}} R_{N}}{\partial \varphi^{\gamma_{j}}}(z, \varphi)
$$

Let us denote by $\nu_{1}$ the number of indices $j \in\{1, \cdots, l(\gamma)\}$ such that $\gamma_{j}=1$, by $\nu_{2}$ the number of indices $j \in\{1, \cdots, l(\gamma)\}$ such that $\gamma_{j}=2 ; \nu_{o}$ is the number of indices $j$ such that $\gamma_{j} \geq 3$ is odd and $\nu_{e}$ is the number of indices such that $\gamma_{j} \geq 4$ is even.

Thus, we have the following two relations:

$$
\nu_{1}+\nu_{2}+\nu_{o}+\nu_{e}=l(\gamma)
$$

and

$$
m=\gamma_{1}+\cdots+\gamma_{l(\gamma)} \geq \nu_{1}+2 \nu_{2}+3 \nu_{o}+4 \nu_{e}
$$

We infer from equation (3.38) the estimate

$\left|R_{N}^{k-l(\gamma)}(z, \varphi) \prod_{j=1}^{l(\gamma)} \frac{\partial^{\gamma_{j}} R_{N}}{\partial \varphi^{\gamma_{j}}}(z, \varphi)\right| \leq C z^{\alpha} \varphi^{4 k-4 l(\gamma)+3 \nu_{1}+2 \nu_{2}+\nu_{o}}$

where $\alpha=k-l(\gamma)+\nu_{1}+\nu_{2}+\nu_{o}+\nu_{e}$, and from equation (3.40), we infer the estimate

$$
\left|R_{N}^{k-l(\gamma)}(z, \varphi) \prod_{j=1}^{l(\gamma)} \frac{\partial^{\gamma_{j}} R_{N}}{\partial \varphi^{\gamma_{j}}}(z, \varphi)\right| \leq C z^{k} \varphi^{4 k-4 l(\gamma)+3 \nu_{1}+2 \nu_{2}+\nu_{o}} .
$$

Equations (3.40) and (3.41) lead to

$$
4 k-4 l(\gamma)+3 \nu_{1}+2 \nu_{2}+\nu_{o}=4 k-\nu_{1}-2 \nu_{2}-3 \nu_{o}-4 \nu_{e} \geq 4 k-m
$$


and the expression $4 k-4 l(\gamma)+3 \nu_{1}+2 \nu_{2}+\nu_{o}$ is also non negative since $l(\gamma)$ belongs to $\{0, \cdots, k\}$; this completes the proof of estimate (3.37). $\mathrm{u}$

We deduce easily an analogous lemma for the derivatives of the powers of $r_{N}$, defined at equation (3.14).

Lemma 3.8. For all $k \in \mathbb{N}^{*}$ and $m \in \mathbb{N}$, there exists $C>0$ such that for all $N \geq 2$, for all $z \in[\pi K, \pi \Lambda N]$ and for all $\varphi \in[0, \pi / 2]$,

$$
\left|\frac{\partial^{m} r_{N}^{k}}{\partial \varphi^{m}}(z, \varphi)\right| \leq C z^{k} \min \left(1, \varphi^{4 k-m}\right) .
$$

Proof. The estimates for $\partial^{m} r_{N} / \partial \varphi^{m}$ are analogous to the estimates (3.38) for $\partial^{m} R_{N} / \partial \varphi^{m}$ and consequently the estimate for $\partial^{m} r_{N}^{k} / \partial \varphi^{m}$ is the same as estimate (3.37) for $\partial^{m} R_{N}^{k} / \partial \varphi^{m}$.

Recall that $\mathcal{I}_{N, \lambda}$ has been defined at equation (3.11). The following corollary gives estimates of its derivatives.

Corollary 3.9. For all integer $k \geq 1$ and all $\lambda \geq 1 / 2$, there exists $C$ such that for all $N \geq 2$ and for all $z$ in $[\pi K, \pi \Lambda N]$,

$$
\max _{s \in[0,1]}\left|\frac{\partial^{k} \mathcal{I}_{N, \lambda}}{\partial s^{k}}(z, s)\right| \leq C\left(N^{-1}+z^{-1}\right)^{k} .
$$

Proof. The derivatives of $\mathcal{I}_{N, \lambda}$ are given by formula (3.13) and we use Lemma 3.5 with $u(z, \varphi)=\psi(\varphi) R_{N}^{k}(z, \varphi) \sin ^{2 \lambda-1} \varphi$ and $b=\pi / 2$; since, in virtue of Lemma 3.7, $\|u\|_{2 k, 4 k, k}$ is finite, we obtain from Lemma 3.5

$$
\max _{s \in[0,1]}\left|\frac{\partial^{k} \mathcal{I}_{N, \lambda}}{\partial s^{k}}(z, s)\right| \leq C\|u\|_{2 k, 4 k, k}\left(N^{-1}+z^{-1}\right)^{k}
$$

that is estimate (3.43).

We estimate in next lemma the derivatives of the difference between $R_{N}^{l}$ and $r_{N}^{l}$, where $r_{N}$ is defined at (3.14).

Lemma 3.10. For all $l \in \mathbb{N}^{*}$ and $m \in \mathbb{N}$, there exists $C>0$ such that for all $N \geq 2$, for all $z \in[\pi K, \pi \Lambda N]$ and for all $\varphi \in[0, \pi / 2]$

$$
\left|\frac{\partial^{m}}{\partial \varphi^{m}}\left(R_{N}^{l}-r_{N}^{l}\right)(z, \varphi)\right| \leq C z^{l} \min \left(1, \varphi^{2 k+4 l-m}\right) .
$$

Proof. First, as in Lemma 3.7, we consider the case $l=1$ and we estimate the successive derivatives of $R_{N}-r_{N}$. We observe that the derivative of order $m$ of $r_{N}$ vanishes for $m \geq 2 k+3$. We calculate the derivatives of $R_{N}-r_{N}$ in terms of the derivatives of $g_{N}$ and using Taylor's formula and Lemma 3.3 we find the inequalities

$$
\begin{aligned}
& \left|\frac{\partial^{m}\left(R_{N}-r_{N}\right)}{\partial \varphi^{m}}(z, \varphi)\right| \leq C \varphi^{2 k+4-m} z, \text { for } m \in\{0, \cdots, 2 k+2\} \\
& \left|\frac{\partial^{m}\left(R_{N}-r_{N}\right)}{\partial \varphi^{m}}(z, \varphi)\right| \leq C \varphi z, \text { for } m \geq 2 k+3, m \text { odd }, \\
& \left|\frac{\partial^{m}\left(R_{N}-r_{N}\right)}{\partial \varphi^{m}}(z, \varphi)\right| \leq C z, \text { for } m \geq 2 k+4, m \text { even. }
\end{aligned}
$$


We factorize $R_{N}^{l}-r_{N}^{l}$ as

$$
R_{N}^{l}-r_{N}^{l}=\left(R_{N}-r_{N}\right)\left(R_{N}^{l-1}+R_{N}^{l-2} r_{N}+\cdots+r_{N}^{l-1}\right)
$$

and a Leibniz formula gives

$$
\begin{aligned}
\frac{\partial^{m}\left(R_{N}^{l}-r_{N}^{l}\right)}{\partial \varphi^{m}}(z, \varphi)=\sum_{\gamma=0}^{m} & \sum_{\beta=0}^{\gamma} \sum_{\nu=0}^{l-1}\left(\begin{array}{c}
m \\
\gamma
\end{array}\right)\left(\begin{array}{l}
\gamma \\
\beta
\end{array}\right) \frac{\partial^{m-\gamma}\left(R_{N}-r_{N}\right)}{\partial \varphi^{m-\gamma}}(z, \varphi) \\
& \times \frac{\partial^{\gamma-\beta} R_{N}^{l-1-\nu}}{\partial \varphi^{\gamma-\beta}}(z, \varphi) \frac{\partial^{\beta} r_{N}^{\nu}}{\partial \varphi^{\beta}}(z, \varphi) .
\end{aligned}
$$

Let us write

$$
T_{\beta, \gamma, \nu}=\frac{\partial^{m-\gamma}\left(R_{N}-r_{N}\right)}{\partial \varphi^{m-\gamma}}(z, \varphi) \frac{\partial^{\gamma-\beta} R_{N}^{l-1-\nu}}{\partial \varphi^{\gamma-\beta}}(z, \varphi) \frac{\partial^{\beta} r_{N}^{\nu}}{\partial \varphi^{\beta}}(z, \varphi) .
$$

Thanks to estimate (3.45), Lemmas 3.7 and 3.8, we can estimate $T_{\beta, \gamma, \nu}$ as follows:

$$
\begin{aligned}
\left|T_{\beta, \gamma, \nu}\right| & \leq C z^{l} \min \left(1, \varphi^{2 k+4-m+\gamma}\right) \min \left(1, \varphi^{4 l-4-4 \nu-\gamma+\beta}\right) \min \left(1, \varphi^{4 \nu-\beta}\right) \\
& \leq C z^{l} \min \left(1, \varphi^{2 k+4 l-m}\right)
\end{aligned}
$$

which proves estimate (3.44).

We can now infer from Lemma 3.10 an estimate of the remainder (3.15):

Corollary 3.11. For $k$ in $\mathbb{N}^{*}, l \in\{0, \cdots, k-1\}$ and $\lambda \geq 1 / 2$, there exists $C>0$ such that for all $N \geq 2$ and for all $z \in[\pi K, \pi \Lambda N]$

$$
\begin{gathered}
\left|\int_{0}^{\pi / 2} \psi(\varphi)\left(R_{N}^{l}(z, \varphi)-r_{N}^{l}(z, \varphi)\right) \exp q_{N}(z, \varphi) \sin ^{2 \lambda-1} \varphi d \varphi\right| \\
\leq C\left(N^{-1}+z^{-1}\right)^{k} .
\end{gathered}
$$

Proof. We set $u(z, \varphi)=\psi(\varphi)\left(R_{N}^{l}(z, \varphi)-r_{N}^{l}(z, \varphi)\right) \sin ^{2 \lambda-1} \varphi$ and $b=\pi / 2$. We deduce from Lemma 3.10 that $\|u\|_{k+l, 2 k+4 l, l}$ is finite and Lemma 3.5 yields equation (3.47).

We state for the reader's convenience the one-dimensional version of Lemma 7.7.3 of Hörmander [12]:

Lemma 3.12. Assume $a \neq 0$ with $\operatorname{Im}(a) \geq 0$ and $u \in \mathcal{S}$, the Schwartz space over $\mathbb{R}$. Then for every $p \in \mathbb{N}^{*}$, there exists $C>0$ such that

$$
\left|\int u(x) e^{i a x^{2} / 2} d x-\left(\frac{a}{2 \pi i}\right)^{-1 / 2} T_{p}(u, a)\right| \leq C\left(\frac{1}{|a|}\right)^{p+1 / 2}\|u\|_{H^{2 p+1}},
$$

with

$$
T_{p}(u, a)=\sum_{j=0}^{p-1} \frac{(2 i a)^{-j}}{j !} \frac{\partial^{2 j} u}{\partial \varphi^{2 j}}(0) .
$$

Here, the principal determination of the fractional power is chosen.

We estimate the last remainder; the number $\chi_{N}$ is defined at equation (1.13). Let $\mathbf{1}_{[a, b]}$ be the characteristic function of $[a, b]$. 
Corollary 3.13. Let $k$ in $\mathbb{N}^{*}, l \in\{0, \cdots, k-1\}$ and $\lambda$ such that $2 \lambda-1$ is an even integer, there exists $C$ such that for all $N \geq 2$, for all $z \in[\pi K, \pi \Lambda N]$,

$$
\begin{aligned}
& \mid \int_{0}^{\pi / 2} \psi(\varphi) r_{N}^{l}(z, \varphi) \sin ^{2 \lambda-1} \varphi e^{\chi_{N} \varphi^{2} / 2} d \varphi-\frac{i}{2} \sqrt{\frac{2 \pi}{\chi_{N}}} \sum_{j=0}^{k+l-1} \frac{1}{j !} \frac{1}{\left(2 \chi_{N}\right)^{j}} \\
& \times \frac{\partial^{2 j}}{\partial \varphi^{2 j}}\left(\psi(\varphi) r_{N}^{l}(z, \varphi) \sin ^{2 \lambda-1} \varphi \mathbf{1}_{[-\pi / 2, \pi / 2]}(\varphi)\right)(z, 0) \mid \\
& \quad \leq C\left(N^{-1}+z^{-1}\right)^{k+1 / 2} .
\end{aligned}
$$

Here, the principal determination of the square root has been chosen.

Proof. We use Lemma 3.12 with

$$
u(z, \varphi)=\mathbf{1}_{[-\pi / 2, \pi / 2]}(\varphi) \psi(\varphi) r_{N}^{l}(z, \varphi) \sin ^{2 \lambda-1} \varphi, p=k+l \text { and } a=-i \chi_{N},
$$

the remainder is equal to $C\left|\chi_{N}\right|^{-(k+l+1 / 2)}\|u\|_{H^{2 p+1}}$.

In virtue of Lemma 3.8, the norm $\|u\|_{2 k+2 l+1,4 l, l}$ is finite and the remainder is bounded by

$$
C\|u\|_{2 k+2 l+1,4 l, l} z^{l}\left(N^{-1}+z^{-1}\right)^{k+l+1 / 2}
$$

which completes the proof.

Now that Lemmas 3.2 to 3.13 are proved, we can apply the strategy of proof described at the beginning of the present section to find an asymptotic formula for $P_{N}^{(\lambda)}$.

TheOREm 3.14. Let $\lambda=1 / 2,3 / 2,5 / 2,7 / 2, \cdots$. Then, there exist real polynomials $Q_{\nu, \lambda}$ of degree $\nu$ for all $\nu \in \mathbb{N}$ such that, for all $k \in \mathbb{N}^{*}$, for all $K \in \mathbb{N}$ and for all $\Lambda \in(0,1 / 2)$, the following estimate holds for all $N \geq 2$ and for all $z \in[\pi K, \pi \Lambda N]$ :

$$
\begin{aligned}
& \mid P_{N}^{(\lambda)}(\cos (z / N)) \\
& -2 \sqrt{\pi} Z(\lambda, N) \operatorname{Re}\left\{i e^{i z} \sum_{\nu=\lambda-1 / 2}^{k-1} \chi_{N}^{-(\nu+1 / 2)} Q_{\nu, \lambda}\left(\chi_{N} / N\right)\right\} \mid \\
& \quad \leq C(K, \Lambda, k, \lambda)\left(N^{-1}+z^{-1}\right)^{k-2 \lambda+1},
\end{aligned}
$$

where $C(K, \Lambda, k, \lambda)$ depends only on the displayed arguments and the constant $Z(\lambda, N)$ is defined at equation (3.1).

Proof. We split (3.3) as in (3.5). Corollary 3.6 implies that the second integral of the right hand side of $(3.5)$ is an $O\left(N^{-1}+z^{-1}\right)^{k}$.

We deduce from equation (3.12) and Corollary 3.9 that

$$
\mathcal{I}_{N, \lambda}(z, 1)=\sum_{l=0}^{k-1} \frac{1}{l !} \frac{\partial^{l} \mathcal{I}_{N, \lambda}}{\partial s^{l}}(z, 0)+O\left(\left(N^{-1}+z^{-1}\right)^{k}\right) .
$$

Let us obtain an expression for

$$
\frac{\partial^{l} \mathcal{I}_{N, \lambda}}{\partial s^{l}}(z, 0)=\int_{0}^{\pi / 2} \psi(\varphi) R_{N}^{l}(z, \varphi) \exp q_{N}(z, \varphi) \sin ^{2 \lambda-1} \varphi d \varphi .
$$


We replace $R_{N}$ by its Taylor expansion $r_{N}$ defined at equation (3.14). We set

$$
\mathcal{J}_{N, l, \lambda}(z)=\int_{0}^{\pi / 2} \psi(\varphi) r_{N}^{l}(z, \varphi) \exp \left(\chi_{N} \varphi^{2} / 2\right) \sin ^{2 \lambda-1} \varphi d \varphi .
$$

Corollary 3.11 implies that

$$
\frac{\partial^{l} \mathcal{I}_{N, \lambda}}{\partial s^{l}}(z, 0)=e^{i z} \mathcal{J}_{N, l, \lambda}(z)+O\left(\left(N^{-1}+z^{-1}\right)^{k}\right) .
$$

We now use Corollary 3.13 to obtain an algebraic expression for $\mathcal{J}_{N, l, \lambda}$. Equation (3.49) yields

$$
\begin{aligned}
\mathcal{J}_{N, l, \lambda}(z)=i & \sqrt{\pi} \sum_{j=0}^{k+l-1} \frac{1}{j !} \frac{1}{\left(2 \chi_{N}\right)^{j+1 / 2}} \frac{\partial^{2 j}\left(r_{N}^{l}(z, \varphi) \sin ^{2 \lambda-1} \varphi\right)}{\partial \varphi^{2 j}}(z, 0) \\
& +O\left(\left(N^{-1}+z^{-1}\right)^{k+1 / 2}\right) .
\end{aligned}
$$

We differentiate $r_{N}^{l}(z, \varphi) \sin ^{2 \lambda-1} \varphi$ with respect to $\varphi$ up to order $2 j$ and we take its value at $\varphi=0$.

Define

$$
s_{n, \lambda}=\frac{\partial^{n} \sin ^{2 \lambda-1}}{\partial \varphi^{n}}(0) .
$$

We first remark that $s_{n, \lambda}$ vanishes when $n$ is odd or $n \leq 2 \lambda-3$. Indeed, since $2 \lambda-1$ is even, $x \mapsto \sin ^{2 \lambda-1} x$ is an even function and its derivatives of odd order at $\varphi=0$ vanish. Moreover, Faa di Bruno's formula (3.17) yields

$$
\begin{aligned}
& \frac{\partial^{n} \sin ^{2 \lambda-1}}{\partial \varphi^{n}}(0)=\sum_{\gamma \in \mathcal{C}(n)} C(\gamma, n)\left(\begin{array}{c}
2 \lambda-1 \\
l(\gamma)
\end{array}\right) l(\gamma) ! \sin ^{2 \lambda-1-l(\gamma)}(0) \\
& \times \prod_{j=1}^{l(\gamma)} \sin \left(\gamma_{j} \frac{\pi}{2}\right) .
\end{aligned}
$$

Consequently, when $n \leq 2 \lambda-3,2 \lambda-1-l(\gamma)$ is positive since $l(\gamma) \leq n$ and thus for all $\gamma \in \mathcal{C}(n), \sin ^{2 \lambda-1-l(\bar{\gamma})}(0)$ vanishes.

Therefore, for $l=0$, we infer that

$$
\mathcal{J}_{N, 0, \lambda}(z)=i \sqrt{\pi} \sum_{j=\lambda-1 / 2}^{k-1} \frac{1}{j !} \frac{1}{\left(2 \chi_{N}\right)^{j+1 / 2}} s_{2 j, \lambda}+O\left(\left(N^{-1}+z^{-1}\right)^{k+1 / 2}\right) .
$$

Consider next the case $l \geq 1$. We need first to calculate the successive even derivatives of $r_{N}^{l}(z, \varphi)$ at $\varphi=0$. We deduce from the definition (3.14) of $r_{N}$ that for $j$ in $\{0, \cdots, 2 l-1\}$ and for $j \geq l(k+1)+1, \partial^{2 j} r_{N}^{l} / \partial \varphi^{2 j}(z, 0)$ vanishes.

Using version (3.17) of Faa di Bruno's formula and observing that for $\gamma$ in $\mathcal{C}(j)$, $r_{N}^{l-l(\gamma)}(z, 0)=\delta_{l, l(\gamma)}$ we find that for $j$ in $\{2 l, \cdots,(k+1) l\}$ :

$$
\frac{\partial^{2 j} r_{N}^{l}}{\partial \varphi^{2 j}}(z, 0)=\sum_{\substack{\gamma \in \mathcal{C}(j) \\ l(\gamma)=l}} C(\gamma, j, l) \prod_{1 \leq i \leq l} \frac{\partial^{2 \gamma_{i}} r_{N}}{\partial \varphi^{2 \gamma_{i}}}(z, 0)
$$


and in virtue of definition (3.14),

$$
=\sum_{\substack{\gamma \in \mathcal{C}(j) \\ l(\gamma)=l}} C(\gamma, j, l) \prod_{1 \leq i \leq l} \frac{\partial^{2 \gamma_{i}} g_{N}}{\partial \varphi^{2 \gamma_{i}}}(z, 0) .
$$

Thanks to equations (3.27), (3.26), (3.28) and (3.25), we obtain

$$
\begin{aligned}
& \frac{\partial^{2 \gamma_{i}} g_{N}}{\partial \varphi^{2 \gamma_{i}}}(z, 0)=(-1)^{\gamma_{i}-1} \chi_{N} \\
& \quad \times \sum_{p=0}^{\gamma_{i}-1}\left(\begin{array}{c}
2 \gamma_{i}-1 \\
2 p
\end{array}\right) \sum_{\alpha \in \mathcal{C}(p)} C(2 \alpha, 2 p) l(\alpha) !\left(\frac{\chi_{N}}{N}\right)^{l(\alpha)},
\end{aligned}
$$

that is to say there exists a real polynomial $T_{\gamma_{i}}$ of degree $\gamma_{i}-1$ such that

$$
\frac{\partial^{2 \gamma_{i}} g_{N}}{\partial \varphi^{2 \gamma_{i}}}(z, 0)=\chi_{N} T_{\gamma_{i}}\left(\chi_{N} / N\right)
$$

Therefore we deduce, from equation (3.54), that for $j$ in $\{2 l, \cdots,(k+1) l\}$,

$$
\frac{\partial^{2 j} r_{N}^{l}}{\partial \varphi^{2 j}}(z, 0)=\chi_{N}^{l} S_{l, j}\left(\chi_{N} / N\right)
$$

where $S_{l, j}$ is a real polynomial of degree $j-l$.

Hence, using Leibniz' formula, we infer for $l \geq 1$ that for $j$ in $\{0, \cdots, k+l-1\}$,

$$
\begin{aligned}
& \frac{\partial^{2 j}\left(r_{N}^{l}(z, \varphi) \sin ^{2 \lambda-1} \varphi\right)}{\partial \varphi^{2 j}}(z, 0)=\chi_{N}^{l} \\
& \times \sum_{m=2 l}^{\min (j-\lambda+1 / 2,(k+1) l)}\left(\begin{array}{c}
2 j \\
2 m
\end{array}\right) s_{2 j-2 m, \lambda} S_{l, m}\left(\chi_{N} / N\right) .
\end{aligned}
$$

Therefore, we deduce that for $j$ in $\{0, \cdots, 2 l+\lambda-3 / 2\}$,

$$
\frac{\partial^{2 j}\left(r_{N}^{l}(z, \varphi) \sin ^{2 \lambda-1} \varphi\right)}{\partial \varphi^{2 j}}(z, 0)=0
$$

and for $j \geq 2 l+\lambda-1 / 2$

$$
\frac{\partial^{2 j}\left(r_{N}^{l}(z, \varphi) \sin ^{2 \lambda-1} \varphi\right)}{\partial \varphi^{2 j}}(z, 0)=\chi_{N}^{l} \tilde{S}_{l, j, \lambda}\left(\chi_{N} / N\right),
$$

where $\tilde{S}_{l, j, \lambda}$ is a real polynomial of degree $j-l-\lambda+1 / 2$.

Eventually, formulas (3.52), (3.57) and (3.58) yield

$$
\mathcal{J}_{N, l, \lambda}(z)=i \sqrt{\pi} \sum_{j=2 l+\lambda-1 / 2}^{k+l-1} \frac{\chi_{N}^{l-j-1 / 2}}{j ! 2^{j+1 / 2}} \tilde{S}_{l, j, \lambda}\left(\chi_{N} / N\right)+O\left(\left(N^{-1}+z^{-1}\right)^{k+1 / 2}\right)
$$

and henceforth

$$
\begin{aligned}
\mathcal{I}_{N, \lambda}(z, 1) & =i \sqrt{\pi} e^{i z} \sum_{l=0}^{k-1} \sum_{j=2 l+\lambda-1 / 2}^{k+l-1} \frac{\chi_{N}^{l-j-1 / 2}}{l ! j ! 2^{j+1 / 2}} \tilde{S}_{l, j, \lambda}\left(\chi_{N} / N\right) \\
& +O\left(\left(N^{-1}+z^{-1}\right)^{k}\right),
\end{aligned}
$$


where $\tilde{S}_{0, j, \lambda}$ is a constant and for $l \geq 1, \tilde{S}_{l, j, \lambda}$ is a polynomial of degree $j-l-\lambda+1 / 2$.

Finally, we obtain with $\nu=j-l$ in formula (3.59) that

$$
\begin{gathered}
P_{N}^{(\lambda)}(\cos (z / N))=Z(\lambda, N) 2 \sqrt{\pi} \operatorname{Re}\left\{i e^{i z} \sum_{\nu=\lambda-1 / 2}^{k-1} \chi_{N}^{-(\nu+1 / 2)} Q_{\nu, \lambda}\left(\chi_{N} / N\right)\right\} \\
+O\left(\left(N^{-1}+z^{-1}\right)^{k-2 \lambda+1}\right),
\end{gathered}
$$

with $Q_{\nu, \lambda}$ of degree $\nu-\lambda+1 / 2$, which completes the proof.

Corollary 3.15 gives explicit values of the asymptotic for the cases $\lambda=1 / 2,3 / 2$, $5 / 2$ and $7 / 2$.

Corollary 3.15. Let $\zeta_{N}=i e^{i z / N} \chi_{N}=N \sin (z / N)$.

For $\lambda=1 / 2$ and $k=3$, Theorem 3.14 yields

$$
\begin{aligned}
& P_{N}^{(1 / 2)}(\cos (z / N))=\sqrt{\frac{2}{\pi}} \frac{1}{\zeta_{N}^{1 / 2}}\left[\cos \left(z+\frac{z}{2 N}+\frac{3 \pi}{4}\right)\right. \\
& \times\left(1-\frac{3}{8 N}+\frac{185}{128 N^{2}}\right)+\frac{1}{\zeta_{N}} \sin \left(z+\frac{3 z}{2 N}+\frac{3 \pi}{4}\right)\left(\frac{1}{8}-\frac{55}{64 N}\right) \\
& \left.-\frac{43}{384} \frac{1}{\zeta_{N}^{2}} \cos \left(z+\frac{5 z}{2 N}+\frac{3 \pi}{4}\right)\right] \\
& +O\left(\left(N^{-1}+z^{-1}\right)^{3}\right) .
\end{aligned}
$$

For $\lambda=3 / 2$ and $k=4$, we obtain

$$
\begin{aligned}
& P_{N}^{(3 / 2)}(\cos (z / N))=-(N+2)(N+1) \sqrt{\frac{2}{\pi}} \frac{1}{\zeta_{N}^{3 / 2}}\left[\cos \left(z+\frac{3 z}{2 N}+\frac{\pi}{4}\right)\right. \\
& \times\left(1-\frac{15}{8 N}+\frac{1505}{128 N^{2}}\right)+\frac{1}{\zeta_{N}} \sin \left(z+\frac{5 z}{2 N}+\frac{\pi}{4}\right)\left(\frac{13}{8}-\frac{735}{64 N}\right) \\
& \left.-\frac{1187}{384 \zeta_{N}^{2}} \cos \left(z+\frac{7 z}{2 N}+\frac{\pi}{4}\right)\right]+O\left(\left(N^{-1}+z^{-1}\right)^{2}\right) .
\end{aligned}
$$

For $\lambda=5 / 2$ and $k=4$, Theorem 3.14 implies

$$
\begin{gathered}
P_{N}^{(5 / 2)}(\cos (z / N))=\frac{1}{18} \sqrt{\frac{2}{\pi}} \frac{(N+4) !}{N !} \frac{1}{\zeta_{N}^{5 / 2}}\left[\cos \left(z+\frac{5 z}{2 N}+\frac{3 \pi}{4}\right)\right. \\
\left.\times\left(6-\frac{105}{4 N}\right)+\frac{115}{4 \zeta_{N}} \sin \left(z+\frac{7 z}{2 N}+\frac{3 \pi}{4}\right)\right]+O(1) .
\end{gathered}
$$

For $\lambda=7 / 2$ and $k=4$, we find

$$
\begin{aligned}
P_{N}^{(7 / 2)}(\cos (z / N))=- & \frac{4}{15} \sqrt{\frac{2}{\pi}} \frac{(N+6) !}{N !} \frac{1}{\zeta_{N}^{7 / 2}} \cos \left(z+\frac{7 z}{2 N}+\frac{\pi}{4}\right) \\
& +O(N+z)^{2} .
\end{aligned}
$$

Proof. We follow the proof of Theorem 3.14 and we find that for $\lambda=1 / 2$,

$$
Q_{0,1 / 2}\left(\chi_{N} / N\right)=1 / \sqrt{2}
$$


and, for $\nu \geq 1$,

$$
\begin{gathered}
Q_{\nu, 1 / 2}\left(\chi_{N} / N\right)=\sum_{\substack{1 \leq l \leq k-1 \\
2 l \leq j \leq k+l-1 \\
j \leq l=\nu}} \sum_{\substack{j+\cdots+\gamma_{l}=j \\
\gamma_{i} \geq 2}} \frac{(2 j) !}{j ! l !} \frac{1}{2^{j+1 / 2}} \\
\times \prod_{1 \leq i \leq l} \frac{1}{\left(2 \gamma_{i}\right) !}\left(\frac{1}{\chi_{N}} \frac{\partial^{2 \gamma_{i}} g_{N}}{\partial \varphi^{2 \gamma_{i}}}(z, 0)\right) .
\end{gathered}
$$

Let us calculate $Q_{1,1 / 2}$ and $Q_{2,1 / 2}$. We infer from equation (3.55) the following derivatives of $g_{N}$ with respect to $\varphi$ at $\varphi=0$ :

$$
\frac{\partial^{4} g_{N}}{\partial \varphi^{4}}(z, 0)=-\chi_{N}\left(1+3 \frac{\chi_{N}}{N}\right)
$$

and

$$
\frac{\partial^{6} g_{N}}{\partial \varphi^{6}}(z, 0)=\chi_{N}\left(1+15 \frac{\chi_{N}}{N}+30 \frac{\chi_{N}^{2}}{N^{2}}\right) .
$$

We deduce from these derivatives that

$$
Q_{1,1 / 2}\left(\chi_{N} / N\right)=-\frac{1}{8 \sqrt{2}}\left(1+3 \frac{\chi_{N}}{N}\right)
$$

and

$$
Q_{2,1 / 2}\left(\chi_{N} / N\right)=\frac{1}{2 \sqrt{2}}\left(\frac{43}{192}+\frac{55 \chi_{N}}{32 N}+\frac{185 \chi_{N}^{2}}{64 N^{2}}\right),
$$

which give the asymptotic formula (3.60).

We use for $\lambda=3 / 2$ the successive derivatives of the square of the sine function at $\varphi=0$ which are

$$
\frac{\partial^{n} \sin ^{2} \varphi}{\partial \varphi^{n}}(0)= \begin{cases}(-1)^{n / 2+1} 2^{n-1} & \text { when } n \text { is even, } n \geq 2 \\ 0 & \text { when } n \text { is odd or } n=0 .\end{cases}
$$

Therefore, we obtain

$$
\begin{aligned}
Q_{\nu, 3 / 2}\left(\chi_{N} / N\right)= & \frac{1}{2}\left[\frac{(-1)^{\nu+1}}{\nu !} 2^{\nu-1 / 2}\right. \\
& +\sum_{\substack{1 \leq l \leq k-1 \\
2 l+1 \leq j \leq k+l-1 \\
j-l=\nu}} \sum_{\substack{2 l \leq m \leq j-1 \\
\gamma_{1}+\cdots+\gamma_{l}=m \\
\gamma_{i} \geq 2}}\left(\begin{array}{c}
2 j \\
2 m
\end{array}\right) \frac{(-1)^{j+m+1}}{2^{2 m+1 / 2-j}} \frac{(2 m) !}{j ! l !} \\
& \left.\times \prod_{1 \leq i \leq l} \frac{1}{\left(2 \gamma_{i}\right) !}\left(\frac{1}{\chi_{N}} \frac{\partial^{2 \gamma_{i}} g_{N}}{\partial \varphi^{2 \gamma_{i}}}(z, 0)\right)\right],
\end{aligned}
$$

and more precisely, we have the following values:

$$
\begin{aligned}
& Q_{1,3 / 2}\left(\chi_{N} / N\right)=1 / \sqrt{2}, \\
& Q_{2,3 / 2}\left(\chi_{N} / N\right)=-\frac{1}{2 \sqrt{2}}\left(\frac{13}{4}+\frac{15 \chi_{N}}{4 N}\right),
\end{aligned}
$$


and

$$
Q_{3,3 / 2}\left(\chi_{N} / N\right)=\frac{1}{2 \sqrt{2}}\left(\frac{1187}{192}+\frac{735 \chi_{N}}{32 N}+\frac{1505 \chi_{N}^{2}}{64 N^{2}}\right)
$$

which lead to equation (3.61).

We calculate the successive derivatives of the sine function to the power 4 at $\varphi=0$ and we find

$$
\frac{\partial^{n} \sin ^{4} \varphi}{\partial \varphi^{n}}(0)=\left\{\begin{array}{lc}
(-1)^{n / 2}\left(2^{2 n-3}-2^{n-1}\right) & \text { when } n \text { is even, } n \geq 4, \\
0 & \text { when } n \text { is odd or } \\
& n=0, n=2 .
\end{array}\right.
$$

These derivatives enable us to calculate:

$$
Q_{2,5 / 2}\left(\chi_{N} / N\right)=6 \sqrt{2}
$$

and

$$
Q_{3,5 / 2}\left(\chi_{N} / N\right)=-\sqrt{2}\left(\frac{115}{4}+\frac{105}{4} \frac{\chi_{N}}{N}\right)
$$

and this yields formula (3.62).

Eventually, the successive derivatives of the sine function to the power 6 at $\varphi=0$ are

$$
\frac{\partial^{n} \sin ^{6} \varphi}{\partial \varphi^{n}}(0)=\left\{\begin{array}{c}
(-1)^{n / 2+1} 2^{n-5} 3\left(3^{n-1}-2^{n+1}+5\right) \\
\text { when } n \text { is even, } n \geq 6, \\
0 \text { when } n \text { is odd or } n=0, n=2, n=4 .
\end{array}\right.
$$

Therefore we find that

$$
Q_{3,7 / 2}\left(\chi_{N} / N\right)=30 \sqrt{2}
$$

and the calculation of formula (3.63) completes the proof.

3.4. Asymptotics of the zeroes of the first derivatives of Legendre polynomials. Now that the formulas for $L_{N}$ and its derivatives have been computed in Corollary 3.15, we can find an asymptotic formula for the zeroes of $L_{N}^{\prime}$ in the region $K \leq k \leq \Lambda N$.

Theorem 3.16. Define

$$
z_{0, k}=\frac{\pi / 4+k \pi}{1+3 / 2 N} .
$$

Then for all $\Lambda$ in $(0,1 / 2)$ and for all $K \in \mathbb{N}$, there exist $C, C^{\prime}$ such that for all $N \geq 2$ and for all integer $k$ in $\{K, \cdots,\lfloor\Lambda N\rfloor\}$, there exists a unique zero $z_{k}$ of $P_{N}^{(3 / 2)}(\cos (z / N))$ in a ball of radius $C^{\prime} / N$ about $z_{0, k}$ and moreover the following estimate holds

$$
\left|z_{k}-z_{0, k}-\frac{13}{8 N \tan \left(z_{0, k} / N\right)}+\frac{22}{3 N^{2} \tan \left(z_{0, k} / N\right)}\right| \leq C\left(N^{-1}+K^{-1}\right)^{3} .
$$


Proof. We use the same method as in the proof of Theorem 2.1 and we use again Lemma 2.2 to calculate an asymptotic formula for the zero $z_{k}$ of $P_{N}^{(3 / 2)}(\cos (z / N))$; this function is given by formula (3.61) of Corollary 3.15.

It is equivalent to calculate the zero $z_{k}$ of

$$
f(z, N)=-\sqrt{\frac{\pi}{2}} \frac{(N \sin (z / N))^{3 / 2}}{(N+2)(N+1)} P_{N}^{(3 / 2)}(\cos (z / N)) .
$$

We are searching this zero in the neighborhood of

$$
z_{0, k}=\frac{\pi / 4+k \pi}{1+3 / 2 N}
$$

We calculate $f\left(z_{0, k}, N\right)$ thanks to formula (3.61) of Corollary 3.15 and we obtain

$$
f\left(z_{0, k}, N\right)=\frac{(-1)^{k}}{\tan \left(z_{0, k} / N\right)}\left(\frac{13}{8 N}-\frac{509}{96 N^{2}}\right)+O\left(\left(N^{-1}+K^{-1}\right)^{5 / 2}\right) .
$$

We differentiate formula (3.68) to obtain:

$$
\begin{aligned}
\frac{\partial f}{\partial z}(z, N) & =\frac{3}{2 N} \frac{f(z, N)}{\tan (z / N)} \\
& +3 \sqrt{\frac{\pi}{2}} \frac{\sqrt{N}}{(N+2)(N+1)} \sin ^{5 / 2}(z / N) P_{N-1}^{(5 / 2)}(\cos (z / N))
\end{aligned}
$$

and using formula (3.69) and equation (3.62) of Corollary 3.15, we find

$$
A(k, N)=\frac{\partial f}{\partial z}\left(z_{0, k}, N\right)=(-1)^{k-1}+O\left(N^{-1}+K^{-1}\right) .
$$

We calculate now the second derivative of the function $f$ using formula (3.70):

$$
\begin{aligned}
& \frac{\partial^{2} f}{\partial z^{2}}(z, N)=\frac{3}{4 N^{2}}\left(\frac{1}{\tan ^{2}(z / N)}-2\right) f(z, N) \\
& +12 \sqrt{\frac{\pi}{2 N}} \frac{N !}{(N+2) !} \cos (z / N) \sin ^{3 / 2}(z / N) P_{N-1}^{(5 / 2)}(\cos (z / N)) \\
& \quad-15 \sqrt{\frac{\pi}{2 N}} \frac{N !}{(N+2) !} \sin ^{7 / 2}(z / N) P_{N-2}^{(7 / 2)}(\cos (z / N)) .
\end{aligned}
$$

Let $C$ be a positive real such that $\left|A^{-1}(k, N) f\left(z_{0, k}, N\right)\right| \leq C N^{-1}$. Let $z$ belong to the ball of center $z_{0, k}$ and of radius $2 C N^{-1}$. We still use formula (3.69) and equations (3.62) and (3.63) of Corollary 3.15 to compute

$$
\frac{\partial^{2} f}{\partial z^{2}}(z, N)=O\left(N^{-1}+K^{-1}\right)
$$

Therefore the number $M$ of Lemma 2.2 is finite and the number $a$ is equal to 1 . The radius $2 C N^{-2}$ has been chosen so that the hypothesis $\left|A^{-1}(k, N) f\left(z_{0, k}, N\right)\right| \leq K$ is satisfied. Therefore, we have the following asymptotic formula:

$$
z_{k}=z_{0, k}+\frac{13}{8 N \tan \left(z_{0, k} / N\right)}+O\left(\left(N^{-1}+K^{-1}\right)^{2}\right) .
$$


In order to have a more precise asymptotic formula, we use once more Lemma 2.2 with the same function $f$ but in the neighborhood of

$$
z_{1, k}=z_{0, k}+\frac{13}{8 N \tan \left(z_{0, k} / N\right)}
$$

We compute the values of $f$ and its derivatives at $z=z_{1, k}$ and we find

$$
\begin{aligned}
f\left(z_{1, k}, N\right) & =(-1)^{k+1} \frac{22}{3 N^{2} \tan \left(z_{0, k} / N\right)}+O\left(\left(N^{-1}+K^{-1}\right)^{3}\right), \\
\frac{\partial f}{\partial z}\left(z_{1, k}, N\right) & =(-1)^{k+1}+O\left(N^{-1}+K^{-1}\right)
\end{aligned}
$$

and if $z$ belongs to the ball of center $z_{1, k}$ and of radius $2 C N^{-1}$, the following estimate holds:

$$
\frac{\partial^{2} f}{\partial z^{2}}(z, N)=O\left(N^{-1}+K^{-1}\right)
$$

Eventually, we obtain the following asymptotic formula

$$
z_{k}=z_{0, k}+\frac{13}{8 N \tan \left(z_{0, k} / N\right)}-\frac{22}{3 N^{2} \tan \left(z_{0, k} / N\right)}+O\left(\left(N^{-1}+K^{-1}\right)^{3}\right) .
$$

We then have the straightforward corollary:

Corollary 3.17. Define

$$
\theta_{0, k}=\frac{\pi(N-k+1 / 4)}{N+1 / 2} .
$$

Then for all $K>0$ and for all $\Lambda \in(0,1 / 2)$, there exist $C, C^{\prime}$ such that for all $N \geq 2$ and for all integer $k$ in $\{K, \cdots,\lfloor\Lambda N\rfloor\}$, there exists a unique zero $\theta_{k}$ of $L_{N}^{\prime}(\cos \theta)$ in a ball of radius $C^{\prime} / N^{2}$ about $\theta_{0, k}$; moreover the following estimate holds

$$
\left|\theta_{k}-\theta_{0, k}-\frac{13}{8 N^{2} \tan \theta_{0, k}}+\frac{49}{12 N^{3} \tan \theta_{0, k}}\right| \leq C\left(\left(N^{-1}+K^{-1}\right)^{4}\right) .
$$

REMARK 3.18. Observe that (3.71) is compatible with (2.1), because the error term in (3.71) is large with respect to the error term in (2.1).

We end this section with the following corollary, which gives the expansion of the quantity $\sigma_{k}$ :

Corollary 3.19. The quantities $\sigma_{k}, K \leq k \leq\lfloor\Lambda N\rfloor$ defined at equation (1.6) have the following expansion:

$$
\sigma_{k}=1+\frac{2}{3 N^{2}}+\frac{\pi^{2}}{6 N^{2}}+\frac{49}{12 N^{2} \tan \left(\eta_{0, k}\right)^{2}}+O\left(\left(N^{-1}+K^{-1}\right)^{3}\right) .
$$

Proof. The proof of this corollary follows the same sketch as the proof of Corollary 2.4. Using equation (3.71) of Corollary 3.17, we find that

$$
\begin{aligned}
L_{N}\left(\xi_{k}\right) & =(-1)^{N+k+1} \sqrt{\frac{2}{\pi}} \frac{1}{\sqrt{N \sin \eta_{0, k}}}\left(1-\frac{1}{4 N}+\frac{67}{96 N^{2}}-\frac{49}{24 N^{2} t^{2}}\right) \\
& +O\left(\left(N^{-1}+K^{-1}\right)^{3}\right)
\end{aligned}
$$


and we use equation (1.3) to compute $1 / \rho_{k}$ :

$$
\frac{1}{\rho_{k}}=\frac{N}{\pi \sin \eta_{0, k}}\left(1+\frac{1}{2 N}+\frac{23}{24 N^{2}}-\frac{49}{12 N^{2} t^{2}}\right)+O\left(\left(N^{-1}+K^{-1}\right)^{2}\right) .
$$

Now, to calculate $\sigma_{k}$, we compute $\xi_{k+1}-\xi_{k-1}$ :

$$
\begin{gathered}
\xi_{k+1}-\xi_{k-1}=2 \sin \eta_{0, k} \frac{\pi}{N}\left(1-\frac{1}{2 N}-\frac{11}{8 N^{2}}-\frac{\pi^{2}}{6 N^{2}}\right) \\
+O\left(\left(N^{-1}+K^{-1}\right)^{4}\right)
\end{gathered}
$$

and we obtain

$$
\sigma_{k}=\frac{2 \rho_{k}}{\xi_{k+1}-\xi_{k-1}}=1+\frac{2}{3 N^{2}}+\frac{\pi^{2}}{6 N^{2}}+\frac{49}{12 N^{2} t^{2}}+O\left(\left(N^{-1}+K^{-1}\right)^{3}\right) .
$$

\section{REFERENCES}

[1] A. Averbuch, A. Cohen, And M. Israeli, A stable and accurate explicit scheme for parabolic evolution equations, http://www.ann.jussieu.fr/ cohen/para.ps.gz,1998.

[2] Christine Bernardi and Yvon Maday, Approximations spectrales de problèmes aux limites elliptiques, Springer-Verlag, Paris, 1992.

[3] Claudio Canuto and Alfio Quarteroni, Preconditioned minimal residual methods for Chebyshev spectral calculations, J. Comput. Phys., 60:2 (1985), pp. 315-337.

[4] Claudio Canuto, Stabilization of spectral methods by finite element bubble functions, Comput. Methods Appl. Mech. Engrg., 116(1-4) (1994), pp. 13-26, ICOSAHOM'92 (Montpellier, 1992).

[5] Mark H. Carpenter, David Gottlieb, and Chi-Wang Shu, On the conservation and convergence to weak solutions of global schemes, J. Sci. Comput., 18:1 (2003), pp. 111-132.

[6] Piero de Mottoni and Michelle Schatzman, The Thual-Fauve pulse: Skew stabilization, Technical Report 304, Équipe d'Analyse Numérique de Lyon, 1999. http://numerix. univ-lyon1.fr/publis/publiv/1999/schatz1609/publi.ps.gz.

[7] M. Deville And E. Mund, Chebyshev pseudospectral solution of second-order elliptic equations with finite element preconditioning, J. Comput. Phys., 60:3 (1985), pp. 517-533.

[8] M. O. Deville And E. H. Mund, Finite-element preconditioning for pseudospectral solutions of elliptic problems, SIAM J. Sci. Statist. Comput., 11:2 (1990), pp. 311-342.

[9] Luigi GatTeschi, Uniform approximation of Christoffel numbers for Jacobi weight, in Numerical integration, III (Oberwolfach, 1987), volume 85 of Internat. Schriftenreihe Numer. Math., pages 49-59. Birkhäuser, Basel, 1988.

[10] E. Hairer, S. P. Nørsett, And G. Wanner, Solving ordinary differential equations. I, volume 8 of Springer Series in Computational Mathematics, Springer-Verlag, Berlin, second edition, 1993, Nonstiff problems.

[11] P. Haldenwang, G. Labrosse, S. Abboudi, and M. Deville, Chebyshev 3-D spectral and 2-D pseudospectral solvers for the Helmholtz equation, J. Comput. Phys., 55:1 (1984), pp. $115-128$.

[12] Lars Hörmander, The analysis of linear partial differential operators. I. Springer Study Edition. Springer-Verlag, Berlin, second edition, 1990, Distribution theory and Fourier analysis.

[13] Steven A. Orszag, Spectral methods for problems in complex geometries, J. Comput. Phys., 37:1 (1980), pp. 70-92.

[14] Seymour V. PARTer, On the Legendre-Gauss-Lobatto points and weights, J. Sci. Comput., 14:4 (1999), pp. 347-355.

[15] Seymour V. PARTer, Preconditioning Legendre special collocation methods for elliptic problems. I. Finite difference operators, SIAM J. Numer. Anal., 39:1 (2001), pp. 330-347 (electronic).

[16] Seymour V. Parter, Preconditioning Legendre spectral collocation methods for elliptic problems. II. Finite element operators, SIAM J. Numer. Anal., 39:1 (2001), pp. 348-362 (electronic). 
[17] Seymour V. Parter and Ernest E. Rothman, Preconditioning Legendre spectral collocation approximations to elliptic problems, SIAM J. Numer. Anal., 32:2 (1995), pp. 333-385.

[18] Alfio Quarteroni and Elena Zampieri, Finite element preconditioning for Legendre spectral collocation approximations to elliptic equations and systems, SIAM J. Numer. Anal., 29:4 (1992), pp. 917-936.

[19] Magali Ribot, Étude théorique de méthodes numériques pour les systèmes de réactiondiffusion; application à des équations paraboliques non linéaires et non locales, $\mathrm{PhD}$ thesis, Université Claude Bernard - Lyon 1, December 2003, http://math. unice.fr/ ribot/ these.pdf.

[20] GÁBor SzEgŐ, Orthogonal polynomials, American Mathematical Society, Providence, R.I., fourth edition, 1975. American Mathematical Society, Colloquium Publications, Vol. XXIII. 